\title{
ZIP9 is a Druggable Determinant of Sex Differences in Melanoma
}

3 Cristina Aguirre-Portolés ${ }^{1}$, Riley Payne ${ }^{2}$, Aspen Trautz ${ }^{1}$, J. Kevin Foskett ${ }^{2}$, Christopher A.

4 Natale $^{1}$, John T. Seykora ${ }^{1}$, Todd W. Ridky ${ }^{1 *}$

$5{ }^{1}$ Department of Dermatology, Perelman School of Medicine, University of Pennsylvania.

$6 \quad{ }^{2}$ Department of Physiology, Perelman School of Medicine, University of Pennsylvania, 7 Philadelphia, PA. USA.

8 *Correspondence to: ridky@pennmedicine.upenn.edu

\section{$\underline{\text { Abstract }}$}

12 Melanoma and most other cancers occur more frequently, and have worse prognosis, in males

13 compared with females. Though sex steroids are thought to be involved, classical androgen and

14 estrogen receptors are not detectable in most melanomas. Here we show that testosterone

15 promotes melanoma proliferation by activating ZIP9 (SLC39A9), a zinc transporter that is not

16 intentionally targeted by available therapeutics, but is widely expressed in human melanoma.

17 This testosterone activity requires zinc influx, MAPK activation and YAP1 nuclear translocation.

18 We demonstrate that FDA approved inhibitors of the classical androgen receptor also inhibit

19 ZIP9, and thereby antagonize the pro-tumorigenic effects of testosterone in melanoma. In male

20 mice, androgen receptor inhibitors suppressed growth of ZIP9-expressing melanomas, but had no

21 effect on isogenic melanomas lacking ZIP9, nor on melanomas in females. These data suggest

22 that ZIP9 might be effectively targeted in melanoma and other cancers by repurposing androgen

23 receptor inhibitors that are currently approved only for prostate cancer. 


\section{$\underline{\text { Introduction }}$}

31 Cancer incidence and mortality are higher in males than in females in the U.S. and worldwide ${ }^{1,2}$.

32 In the U.S., males are 15\% more likely to develop cancer, and $40 \%$ more likely to die from this

33 disease than females ${ }^{1}$. These sex differences were recognized as early as $1949^{3}$, are observed in

34 the majority of cancer types from non-reproductive tissues ${ }^{1}$, and remain even after controlling for

35 known risk factors such as environmental and occupational exposures ${ }^{4}$. While recent advances in 36 modern targeted and immune therapeutics have markedly improved survival for both female and

37 male melanoma patients ${ }^{5}$, females still have more favorable outcomes ${ }^{1,4}$. Defining the 38 mechanisms underlying the broad and persistent sex differences in cancer incidence and 39 outcomes will address a major unresolved question in cancer pathobiology.

40 We previously showed that the female sex hormone estradiol inhibits melanoma 41 proliferation and tumor growth in vivo. This effect is independent of biologic sex of the tumor,

42 independent of classical estrogen receptors (ER), and results from activation of a nonclassical 43 surface estrogen receptor on melanocytes and melanoma cells called the G Protein Estrogen

44 Receptor (GPER) $)^{6,7}$. This work led us to consider whether male sex hormone signaling might 45 also contribute to female vs. male differences in melanoma progression.

Testosterone is the most abundant androgen and circulates at much higher levels in males

$47(630 \mathrm{ng} / \mathrm{dl})$ than in females $(32 \mathrm{ng} / \mathrm{dl})^{8}$. This androgen promotes proliferation in vitro in non48 gonadal cell types including adipocytes ${ }^{9}$, mouse skeletal muscle myoblasts ${ }^{10}$, glioblastoma49 derived cells ${ }^{11}$, lung cancer cell lines ${ }^{12}$ and melanoma ${ }^{13}$. As the classical androgen receptor (AR)

50 is not consistently detected in most of these tissues, the receptor(s) mediating the testosterone51 dependent increased proliferation and the corresponding downstream mechanisms are not yet 
52 defined. Also unknown is whether this androgen activity observed in vitro is relevant to cancer 53 progression in vivo. Here, we define a nonclassical testosterone signaling pathway that establishes a novel mechanistic link between male androgens and melanoma behavior, and highlights a new therapeutic opportunity using currently-available drugs.

\section{$\underline{\text { Results }}$}

Melanoma tumors grow more quickly in male vs. female mice

To test whether preclinical melanoma models recapitulate the male vs. female survival disparity observed in humans, we first determined growth of human BRAF-driven melanoma (WM46, female origin, BRaf ${ }^{\mathrm{V} 600 \mathrm{E}}$; $\mathrm{CDK} 4^{\mathrm{R} 24 \mathrm{C}}$ ). Tumors grew faster in immunodeficient SCID male mice compared with matched female mice (Fig. 1A and Fig. S1A), indicating that the differences do not depend on $\mathrm{T}$ and/or $\mathrm{B}$ cell immune responses. To test whether this phenotype extends to a genetically-engineered preclinical mouse model, we used murine YUMM1.7 cells (male origin, $\mathrm{BRaf}^{\mathrm{V} 600 \mathrm{E} / \mathrm{wt}}$; Pten $\left.{ }^{-/-} \mathrm{CdkN} 2 \mathrm{a}^{-/-}\right)^{14}$ in syngeneic immunocompetent $\mathrm{C} 57 \mathrm{BL} / 6$ mice. These tumors also consistently grew faster in male mice compared with matched females (Fig. S1B).

Testosterone, but not dihydrotestosterone, promotes proliferation in melanoma cells independent of the classical androgen receptor response to testosterone correlated to the dose and was saturable, suggesting a specific receptormediated activity. In contrast, dihydrotestosterone (DHT) had no effect on proliferation of these 
cell types across a wide concentration range (Fig. 1C). Dihydrotestosterone is a more potent AR agonist than testosterone ${ }^{15,16}$. The fact that the melanocytes and melanoma cells responded to testosterone, but not to DHT (Fig. 1B, 1C, Fig S1D, E), suggests the possibility that a

80 nonclassical androgen receptor mediates testosterone effects in these cells. Supporting this idea,

81 we were unable to detect AR mRNA transcript in any of the 7 melanoma cell lines (Table S1)

82 and primary melanocyte (MC) cultures we tested, although AR mRNA was readily detectable in

83 prostate cancer cells (Fig. 1D, Fig. S1F). Consistent with this lack of AR transcript, we were also

84 unable to detect AR protein in these cells via western blotting using 2 different AR antibodies, or

85 via immunofluorescence directly on the cultured cells, although AR protein was readily

86 detectable in 2 prostate cancer lines used as positive controls (Fig. 1E and Fig. S1G).

87 To begin testing whether a nonclassical androgen receptor mediates testosterone effects

88 in melanoma we looked to SLC39A9/ZIP9, a transmembrane zinc transporter recently discovered

89 in Atlantic Croaker (fish) cells to be a activated by androgen, and unlike AR, to have much

90 higher affinity for testosterone than for dihydroteststerone ${ }^{17}$. ZIP9 transports zinc $\left(\mathrm{Zn}^{++}\right)$across

91 cell and organelle membranes into the cytoplasm and is the only steroid receptor known to

92 directly regulate zinc homeostasis ${ }^{18}$. The human ZIP9 homolog, encoded by the SLC39A9 gene,

93 is broadly expressed in most cancers (Fig. S2A), normal human melanocytes, and all melanoma

94 lines that we tested (Fig. 2A). To test whether ZIP9 was active in melanoma we used the

95 fluorescent $\mathrm{Zn}^{++}$-specific probe FluoZin-3. Testosterone induced a rapid increase in cytosolic

96 free $\mathrm{Zn}^{++}\left(\left[\mathrm{Zn}^{++}\right]_{\mathrm{i}}\right)$ in human melanoma cells (Fig. 2B), that was followed by a sustained

97 elevation for at least 2 days in the presence f testosterone (Fig. 2C). We determined that $\mathrm{Zn}^{++}$

98 influx was necessary for the testosterone-dependent increase in melanoma cell proliferation, as

99 treatment with the $\mathrm{Zn}^{++}$chelator $\mathrm{N}, \mathrm{N}, \mathrm{N}^{\prime}, \mathrm{N}^{\prime}$-tetrakis (2-pyridinylmethyl)-1,2-ethanediamine 
100 (TPEN), blocked testosterone induced proliferation at TPEN concentrations that had no 101 significant effect on proliferation when used alone (Fig. 2D and Fig. S2B).

Although TPEN has high affinity for $\mathrm{Zn}^{++}$, it also has some ability to chelate copper ${ }^{19}$. To

103 test whether copper flux might contribute to testosterone effects on melanoma proliferation, we

104 treated WM46 cells with $\mathrm{CuSO}_{4}$, which had no significant effect on cell proliferation (Fig. S2C).

105 Moreover, the highly specific copper chelator bathocuproinedisulfonic acid (BCS), did not affect

106 the proliferative response to testosterone, nor did BCS affect testosterone induced $\mathrm{Zn}^{++}$influx

107 (Fig. S2D and Fig. S2E).

After determining that $\mathrm{Zn}^{++}$influx was necessary for testosterone-induced proliferation,

109 we next tested whether increased $\mathrm{Zn}^{++}$was sufficient to increase melanoma proliferation.

110 Exogenous zinc pyrithione (also a $\mathrm{Zn}^{++}$ionophore), and $\mathrm{ZnCl}_{2}$ each increased melanoma

111 proliferation (Fig. 2E). Other biologically-relevant divalent cations, $\mathrm{Fe}^{++}$and $\mathrm{Mn}^{++}$, known to be

112 transported by other ZIP family members ${ }^{20}$, had no effect on proliferation (Fig. 2E). Together,

113 these results demonstrate that testosterone, but not DHT, promotes zinc dependent proliferation

114 in melanoma cells that express ZIP9 and lack detectable AR.

115 We next used immunocytochemistry to test whether AR and/or ZIP9 protein are

116 expressed in human melanocytic lesions (14 benign nevi, 63 primary melanomas, and 21

117 metastatic melanomas). AR was determined in tissue sections using a highly validated CLIA

118 (Clinical Laboratory Improvement Amendments) certified method in the clinical pathology lab at

119 the Hospital of the University of Pennsylvania. This procedure uses a validated antibody

120 different from the two we used for western blotting and immunofluorescence. Each tissue was

121 scored in a blinded fashion by a board-certified pathologist. While AR was readily detectable in

122 prostate tissue used as positive control, AR was not detected in any of the nevi, nor in the 
123 melanomas (Fig. 2F). In parallel, we analyzed the same samples for ZIP9. CLIA grade ZIP9

124 IHC is not available. However, we validated our ZIP9 antibody using parental WM46 ZIP9

125 positive (wtZIP9) and isogenic ZIP9 negative ( $\triangle$ ZIP9) cell lines. These cells were grown on

126 chamber slides and processed for IHC in parallel with the human samples. The antibody used

127 labeled only the wtZIP9 cells. ZIP9 protein was observed in $100 \%$ of the nevi, 97\% of primary

128 melanomas and $100 \%$ of the metastatic samples (Fig. 2G). Further, ZIP9 relative staining

129 intensity positively correlated with tumor stage (Fig. 2H). No significant differences in ZIP9

130 staining were observed between males and females (Fig. S3).

\section{Testosterone signaling through ZIP9 promotes melanoma growth}

133 To determine whether ZIP9 mediates testosterone effects in our models, we used CRISPR-Cas9

134 and gRNA targeting Slc39A9 exon1 to ablate ZIP9 in human (WM46) melanoma and in murine

135 (YUMM1.7) melanoma. Isogenic clonal populations of parental ZIP9-expressing (wtZIP9) and

136 ZIP9-ablated (LZIP9) cells were established, and the mutations disrupting the reading frame

137 were mapped by sequencing (Fig. S4A, S4B and S4C). ZIP9 protein was not detectable in $\Delta$ ZIP9

138 cells (Fig. 2F, Fig. 3A, Fig. 3B and Fig. S4D). $\triangle$ ZIP9 cells did not respond to testosterone,

139 whereas isogenic wtZIP9 control clones responded similarly to the parental wtZIP9 cells (Fig.

$140 \quad 3 \mathrm{~A}, 3 \mathrm{~B}$ and Fig. S4E).

141 The testosterone insensitive phenotype associated with CRISPR-Cas9 engineered ZIP9 loss

142 was rescued by expression of a human ZIP9 transgene. Lentiviral mediated constitutive ZIP9

143 expression in the $\triangle$ ZIP9 WM46 cells restored both ZIP9 protein and the proliferative response to

144 testosterone, verifying the on target effects of the CRISPR-Cas9 mediated ZIP9 ablation (Fig.

145 S4F, S4G). 
Consistent with the conclusion that ZIP9 is the major mediator of testosterone and exogenous zinc effects in these melanoma cells, exposure to testosterone or to extracellular zinc

148 failed to increase $\left[\mathrm{Zn}^{++}\right]_{i}$ in $\triangle \mathrm{ZIP9}$ cells (Fig. 3C and 3D). Therefore, testosterone induces a 149 rapid, ZIP9-dependent, increase in $\left[\mathrm{Zn}^{++}\right]_{\mathrm{i}}$ that is required for the increased proliferation in 150 melanoma cells.

\section{and YAP1 activation}

154 Although the studies detailed above show that testosterone promotes melanoma proliferation via

155 ZIP9 dependent $\mathrm{Zn}^{++}$influx, zinc is involved in myriad cellular processes, making it difficult to 156 predict a priori which downstream signaling pathways are required for the testosterone activity.

157 To start identifying these, we used a 447-element Reverse Phase Protein Array (RPPA) analysis 158 of human WM46 melanoma cells treated with testosterone for $0,30,60$ and 480 min. While the 159 relative expression of most represented proteins was unaffected by testosterone, some were 160 significantly under or overexpressed, including several with known tumor-promoting or tumor161 suppressive functions (Fig. 4A and Table S2). Downregulated proteins included 14-3-3e, a tumor 162 suppressor and negative YAP1 regulator previously implicated in liver, lung, and gastric 163 cancers $^{21}$, and CDKN2A (p16), a cyclin-dependent kinase (CDK) inhibitor, and one of the most 164 studied tumor suppressors ${ }^{22}$. Upregulated proteins included key elements of tumor-promoting 165 pathways, most notably phosphorylated ERK (T202; Y204) and YAP1 (Fig. 4A).

166 Importantly, $\triangle$ ZIP9 cells displayed decreased basal levels of ERK phosphorylation compared 167 with isogenic wild-type clones (Fig. S5A). MAPK activation was necessary for the testosterone168 dependent proliferative response, as the specific ATP-competitive ERK1/2 inhibitor, ulixertinib ${ }^{23}$ 
169 (RVD-523; Ei) blocked the testosterone-dependent proliferative response, at ulixertinib 170 concentrations that had no effect on the basal proliferation rate when used alone (Fig. 4B). 171 Testosterone-dependent ZIP9 activation appeared to render WM46 cells more sensitive to 172 ulixertinib, as cell viability was compromised when they were treated with both testosterone and 173 RVD-523. This vulnerability to combination treatment seems to be specific and ZIP9 dependent, 174 as $\triangle$ ZIP9 cells treated with both testosterone and ulixertinib proliferated at rates comparable to 175 controls (Fig. 4B).

176 We determined that YAP1 activation is also required for the augmented proliferative 177 response driven by testosterone. YAP1 is a transcriptional coactivator whose activity is largely 178 regulated by its localization ${ }^{24}$. Testosterone induced rapid YAP1 translocation from cytoplasm to 179 nucleus in human melanoma cells in a ZIP9-dependent manner (Fig. 4C, 4D and Fig. S5B). 180 YAP1 subcellular localization is controlled largely by LATS, which phosphorylates YAP1 at 181 Serine 127 and thereby retains YAP1 in the cytoplasm ${ }^{25}$. The YAP1 inhibitor dobutamine also 182 promotes phosphorylation of YAP at Ser12726. Consistent with this, the testosterone induced 183 YAP1 nuclear localization and increase in cell proliferation were both blocked by dobutamine, 184 while this compound had no significant effect on its own (Fig. 4D, Fig. S5C and Fig. 4E). When 185 ZIP9 expression was rescued via lentiviral transduction of a ZIP9 transgene to $\Delta$ ZIP9 WM46 186 cells, testosterone dependent YAP1 translocation into the nucleus was also restored (Fig. S5C).

187 Testosterone promotion of YAP1 activity was evidenced by increased expression of 188 several well-known YAP1 target genes including CDC6, CTGF, CYR61 and THBS1. As 189 expected, these testosterone-induced expression changes were also blocked by dobutamine (Fig. $1904 \mathrm{~F})$. 
191 Pharmacologic ZIP9 blockade inhibits testosterone-driven melanoma proliferation and melanoma tumor growth in vivo.

We next tested whether FDA-approved compounds could be repurposed to effectively target ZIP9. Although specific ZIP9 inhibitors are not yet developed, molecular modeling and competition assays with fluorescent-tagged testosterone suggest that the androgen receptor

196 inhibitor bicalutamide (BIC) competes with testosterone for ZIP9 binding to the same 197 extracellular pocket and thereby acts as a competitive ZIP9 antagonist ${ }^{27}$. Bicalutamide was

198 developed and approved for prostate cancer and has now been largely replaced by enzalutamide 199 (ENZ) and apalutamide (APA), which are structurally related analogs with higher affinity for the androgen receptor and greater clinical efficacy against advanced prostate cancer ${ }^{28,29}$.

While ZIP9 was not known to be an androgen receptor at the time these drugs were

202 developed, we show here that they are nonetheless effective inhibitors of testosterone effects in 203 melanoma cells that express ZIP9, but lack detectable AR. Each compound completely blocked 204 testosterone-induced proliferation and MAPK activation in several melanoma cell lines (Fig. 5A, 205 5B). Importantly, these agents alone (without testosterone) had no effect on cell proliferation 206 (Fig. S6A). The testosterone dependent increase of intracellular zinc was also efficiently blocked 207 by bicalutamide (Fig. S6B). Moreover, $\triangle$ ZIP9 cells transduced to re-express ZIP9, responded to 208 testosterone, and this effect was again blocked by bicalutamide (Fig. S6C).

To further confirm that these pharmacologic agents work through ZIP9, we next used 210 cyproterone acetate (CPA), an anti-androgen that blocks the testosterone interaction with AR, but 211 that does not bind to ZIP9 ${ }^{30}$. In our melanoma cells, up to a 20 -fold molar excess of CPA did not 212 significantly inhibit testosterone effects on proliferation, consistent with the conclusion that AR 213 is not the major mediator of testosterone effects in these melanoma models (Fig. S6D). 
To test whether these pharmacologic flutamide class AR inhibitors block the physical

215 interaction between testosterone and ZIP9 in melanoma, we performed direct binding assays

216 using a membrane-impermeable testosterone analogue (T-BSA-FITC). This reagent labels the

217 plasma membrane surface of wild-type ZIP9 expressing WM46 melanoma cells (Fig. 5C). This

218 membrane bound testosterone was displaced by apalutamide, demonstrating the specificity of the

219 interaction (Fig. 5C). Further demonstrating that the binding is ZIP9 specific, testosterone

220 localization at the plasma membrane was markedly reduced in $\triangle$ ZIP9 cells (Fig. 5C) that did not

221 respond to either testosterone, or to APA in vitro (Fig. 5D).

222 Next, we tested whether apalutamide and related analogs inhibit melanoma in vivo. For this, we

223 introduced wtZIP9 or isogenic $\triangle$ ZIP9 human WM46 melanoma into SCID mice. Once-daily

224 systemically administered apalutamide $(20 \mathrm{mg} / \mathrm{kg}$ /day via oral gavage) significantly suppressed

225 growth of wtZIP9 tumors in male mice and extended its survival (doubling time for tumor

226 growth was 11.1 days for vehicle treated males and 25.2 days for APA treated males) (Fig. 5E,

227 Fig. S7A). Similar results were obtained when males were treated with bicalutamide

228 (30mg/kg/day) (Fig. S7B). Importantly, bicalutamide had no effect on wtZIP9 tumors in female

229 mice, nor on $\triangle$ ZIP9 tumors (Fig. S7C and S7C). Together, these data show that ZIP9 promotes

230 melanoma progression specifically in males.

\section{$\underline{\text { Discussion }}$}

Although many have speculated that sex steroids contribute to sex differences in cancer

235 pathobiology, definitive functional studies are lacking, and the mechanisms by which they

236 contribute to the male-female cancer sex gap are largely unknown. We previously described the

237 tumor suppressor activity of the nonclassical estrogen receptor GPER in melanoma ${ }^{6}$. This 
238 finding led us to question whether circulating androgens in males might also contribute to the 239 melanoma sex gap.

Testosterone promoted proliferation of melanoma cells in a saturable-dose-dependent

241 manner. Curiously, the same cell lines did not respond to DHT, which is generally considered

242 the more biologically active androgen. DHT displays 4-fold increased affinity than testosterone

243 for $\mathrm{AR}$, and it dissociates from AR three time slower than testosterone ${ }^{16}$. Lack of response to

244 DHT in melanoma is consistent with the absence of detectable AR expression in the models used

245 for this study, and is highly consistent with our genetic data showing that ZIP9, which has higher

246 affinity for testosterone vs. DHT, is an important mediator of testosterone effects.

247 We show that ZIP9 activation via testosterone binding promotes an increase in cytosolic

248 zinc in melanoma cells. The mammalian family of zinc transporters SLC39A comprises 14

249 members (ZIP1-14) $)^{31}$ grouped into four subfamilies that were stablished according to their

250 amino acid sequence similarities. There may be roles for these other family members in some

251 cancers, as ZIP1 (SLC39A1, from ZIPII subfamily) that has been associated with the regulation

252 of zinc uptake in prostate cancer cells ${ }^{32}$. However, this rapid increase of intracellular zinc

253 through ZIP1 appears to be AR dependent, as PC-3 cells only respond to testosterone after they

254 are transfected with exogenous AR. ZIP9 amino acid residues predicted to be most critical for

255 testosterone and bicalutamide binding include Ala167, Val241, Met248, and Ala167, Leu302,

256 Ser171, respectively ${ }^{27}$, however only Ala167 is present in ZIP1, suggesting that this receptor

257 may not be similarly regulated by testosterone. Regarding other members of the SLC39A family,

258 ZIP9 is unique member of ZIPI subfamily ${ }^{33}$, and the only one known to directly interact with

259 testosterone. 
A recent report $^{34}$ also considered a possible role for testosterone in melanoma and concluded that the androgen promotes melanoma. Authors attributed this effect to the classical AR. However, that report did not consider ZIP9, did not show that AR was necessary for

263 melanoma response to pharmacologic AR inhibitors, and did not show that AR was a 264 determinant of sex differences in melanoma tumor growth. Here we studied 98 human 265 melanocytic lesions (nevus, primary and metastatic melanoma) and did not detect AR in any of 266 them, while ZIP9 protein was detectable in $97 \%$ and $100 \%$ of the primary and metastatic 267 melanomas respectively. We recognize the possibility that some melanoma cell lines and perhaps even some human tumors, may express AR that also contributes to melanoma growth.

There may be many drivers of the cancer sex gap in humans, including differences in 270 immune surveillance ${ }^{35}$. However, differences in immune surveillance do not appear to be a major 271 driver of the differences in the melanoma models used for this study, as tumors progressed faster 272 not only in male vs. female immunocompetent mice, but also in human melanomas grown in 273 male vs. female SCID mice. Therefore, the testosterone effects on melanoma in these models are 274 dependent on ZIP9, but independent of B and T cell mediated anti-tumor activity. Consistent 275 with this, ZIP9 expressing tumors respond to bicalutamide and apalutamide in SCID mice.

As ZIP9 is widely expressed in nearly all tissues, it may be a major determinant of the 277 sex disparity in outcomes not just for melanoma, but also for many other cancer types. 278 Consistent with this, we observed that testosterone promotes proliferation in many diverse cancer 279 cell types, and that this effect is blocked by bicalutamide (Fig. S8).

281 may be a new eminently druggable therapeutic target (Fig. 5F), and that currently approved 282 androgen receptor inhibitors might be useful alone, or in combination with current standard of 
283 care therapeutics for a wide range of cancers, especially those that disproportionately affect

284 males.

\section{Materials and Methods}

\section{CRISPR-Cas9 mediated ablation of Slc39A9}

289 We used lentiviral transduction to deliver dox-inducible Cas9 and gRNA targeting exon 1 of 290 Slc39A9 in human WM46 and murine YUMM1.7 melanoma cells. Transduced cells were

291 selected with puromycin, and single cells subsequently isolated, expanded and examined for 292 ZIP9 protein expression, compared to clones isolated in parallel with no doxycycline treatment.

293 The following gRNA sequences were used (5'-3')':

294 hZIP9_gRNA_Fw caccgTTGGTGGGATGTTACGTGGC

295 hZIP9_gRNA_Rv aaacGCCACGTAACATCCCACCAAC

296 hZIP9_gRNA_Fw caccgCGTGGCCGGAATCATTCC

297 hZIP9_gRNA_Rv aaacGGAATGATTCCGGCCACG

298 To map the targeted sequencing, the region surrounding the gRNA target sequence was 299 amplified in both WM46 (282bp) and YUMM1.7 (259bp) isogenic clones. The following 300 primers were used $\left(5^{\prime}-3^{\prime}\right):$ hZIP9_CRISPRmut_Fw:

301 TAAGCAGAATTCATGGATGATTTCATCTCC

302 hZIP9_CRISPRmut_Rv:TAAGTAAGTCCAAGCTTCTGCTGCTTTGTCTGATGCA.

303 mZIP9_CRISPRmut_F:TAAGCAGAATTCATGGATGACTTTCTCTC.

304 mZIP9_CRISPRmut_Rv TAAGTAAGTCCAAGCTTGATATTTCTGCTGCTTTGT. 
305 Once amplified, DNA fragments were cloned into pUC19 vector using EcoRI and HindDIII

306 sites, and sequenced (Sanger) by the DNA Sequencing Facility (University of Pennsylvania).

307 Sequences were analyzed using the free software CRISP-ID (V1.1) and ICE Analysis by

308 Synthego were the knock-out scores were obtained.

310 Cell culture and proliferation assays

311 YUMM1.7, SH-4 and SK-MEL-2 cells were purchased from ATCC (YUMM1.7 ATCC® CRL-

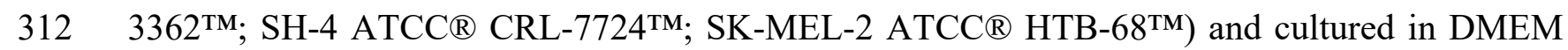

313 (Mediatech, Manassas, VA, USA) with 5\% FBS (Invitrogen, Carlsbad, CA, USA) and 1\%

314 Penicillin-Streptomycin (Thermo Fisher Scientific. \# 15140122). SK-MEL-3 cells were

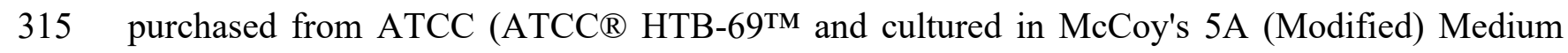
316 with 10\% FBS (Invitrogen, Carlsbad, CA, USA) and 1\% Penicillin-Streptomycin. SK-MEL-24 317 cells were purchased from ATCC (ATCC ${ }^{\circledR}$ HTB-71 ${ }^{\mathrm{TM}}$ ) and cultured in Eagle's Minimum 318 Essential Medium with 15\% FBS and 1\% Penicillin-Streptomycin. WM46 melanoma cells were 319 a gift from Meenhard Herlyn (Wistar Institute, Philadelphia, PA, USA) and were cultured in 320 TU2\% media. Tumor cells were regularly tested using MycoAlert Mycoplasma Detection Kit 321 from Lonza (Allendale, NJ, USA). For monitoring cell proliferation 10x10 ${ }^{5}$ YUMM1.7 or $32212 \times 10^{5}$ WM46 cells were seed per well in 12-well Cell culture Plates. All the experiments 323 performed in this work utilized charcoal stripped serum (Fetal Bovine Serum, charcoal stripped, 324 USDA-approved regions, One Shot ${ }^{\mathrm{TM}}$ format. Catalogue number \#A3382101; Thermofisher 325 Scientific). Cells were treated every second day and manually counted in triplicate using a 326 hemocytometer. All the experiments were performed in cell populations that were in culture 327 during a maximum of 3 weeks ( 5 passages in average) since thaw from the corresponding stock. 


\section{Zinc influx analysis}

329 WM46 cells were loaded with $5 \mu \mathrm{M}$ FluoZin ${ }^{\mathrm{TM}}-3$, AM cell permeant ${ }^{\mathrm{TM}}$ (Thermo Fisher 330 Scientific, \#F24195), for 20 minutes then incubated in 2 Ca Tyrode's solution (in mM: 140

$331 \mathrm{NaCl}, 5 \mathrm{KCl}, 1 \mathrm{MgCl}_{2}, 2 \mathrm{CaCl}_{2}, 10$ glucose and $5(\mathrm{Na})$ Pyruvate $\left.-\mathrm{pH} 7.4\right)$ for 5-10 minutes at 332 room temperature prior to imaging. Cells were imaged on a Nikon Ti microscope using a $33320 x / 0.75 \mathrm{NA}$ objective for fluorescence at $340 \mathrm{~nm}$ and $380 \mathrm{~nm}$ excitation $/ 515 \mathrm{~nm}$ emission $\left(\mathrm{Ca}^{2+}{ }_{-}\right.$ 334 free Fura2 or FluoZin $\left.{ }^{\mathrm{TM}}-3\right)$ and $380 \mathrm{~nm}$ excitation/515 nm emission $\left(\mathrm{Ca}^{2+}\right.$-free Fura2 FluoZin ${ }^{\mathrm{TM}}$ 335 3). Coverslips were perfused at $1-3 \mathrm{~mL} / \mathrm{min}$ following this protocol: $\mathrm{Ca}^{2+}$ Tyrode's $(0-30 \mathrm{secs})$; $\mathrm{Ca}^{2+}$ Tyrode's + DMSO \& 5 uM Zinc (30-90 secs); $\mathrm{Ca}^{2+}$ Tyrode's + Testosterone \& 5 M Zinc (90-360 secs); $\mathrm{Ca}^{2+}$ Tyrode's + Testosterone + BIC \& 5 uM Zn (360-470 secs); $\mathrm{Ca}^{2+}$ Tyrode's washout (470-500 secs). 100 milliseconds exposure images for each wavelength were collected every 2 seconds. For analyzing the long-term consequences of testosterone treatment, cells were

340 treated with the androgen for 96 hours and then loaded FluoZin ${ }^{\mathrm{TM}}-3$. Cells were incubated with 341 FluoZin ${ }^{\mathrm{TM}}-3$ following manufacturer recommendation and exposed to $400 \mathrm{nM} \mathrm{Zn}$ pyrithione or $342 \mathrm{ZnCl}_{2}$. Images were acquired with EVOS BLA. Fluorescence was quantitated using ImageJ 343 (National Institutes of Health, Bethesda, MD, USA), and statistical analyses were performed 344 using Graphpad Prism software.

\section{Reverse Phase Protein Array (RPPA)}

347 We used the Functional Proteomics Core at MD Anderson 348 (https://www.mdanderson.org/research/research-resources/core-facilities/functional-proteomics-

349 rppa-core.html) to perform a 447-element Reverse Phase Protein Array (RPPA) analysis of 350 human melanoma cells grown in medium with stripped serum and treated with testosterone 
351 (100nM) for $0,30^{\prime}, 60^{\prime}$ and 8 hours. Cells were tripsinized and washed with PBS. After

352 centrifugation (5 minutes; $1200 \mathrm{rpm}$ ), supernatant was discarded, and cells were frozen at $-80^{\circ} \mathrm{C}$

353 prior to be sent to the Functional Proteomics Core at MD Anderson.

\section{Western blot, immunofluorescence and antibodies}

356 Adherent cells were washed once with PBS and lysed with $8 \mathrm{M}$ urea containing $50 \mathrm{mM} \mathrm{NaCl}$ and $35750 \mathrm{mM}$ Tris- $\mathrm{HCl}, \mathrm{pH} 8.3,10 \mathrm{mM}$ dithiothreitol, 50mM iodoacetamide. Lysates were quantified

358 (Bradford assay), normalized, reduced, and resolved by SDS gel electrophoresis on 4-15\% 359 Tris/Glycine gels (Bio-Rad, Hercules, CA, USA). Resolved protein was transferred to PVDF 360 membranes (Millipore, Billerica, MA, USA) using a Semi-Dry Transfer Cell (Bio-Rad), blocked 361 in 5\% BSA in TBS-T and probed with primary antibodies recognizing $\beta$-actin (Cell Signaling

362 Technology, \#3700. 1:4000, Danvers, MA, USA), ZIP9 (Abcam, \#137205, 1:500), P-ERK (Cell 363 Signaling Technology, Phospho-p44/42 MAPK (Erk1/2) (Thr202/Tyr204) (D13.14.4E) XP®

364 Rabbit mAb \#4370. 1:1000), ERK (Cell Signaling Technology, p44/42 MAPK (Erk1/2) (137F5)

365 Rabbit mAb \#4695, 1:1000), Androgen Receptor [(D6F11) XP® Rabbit mAb \#5153] and 366 Recombinant Anti-Androgen Receptor antibody [EPR1535(2)] (ab133273. Antibody already

367 validated by the Human Protein Atlas). After incubation with the appropriate secondary antibody 368 [(Rabbit Anti-Mouse IgG H\&L (Biotin) preabsoFS9rbed (ab7074); Anti-mouse IgG, HRP-linked 369 Antibody \#7076. 1:2000)] proteins were detected using ClarityTM Western ECL Substrate (Bio370 Rad. \#170-5060). All western blots were repeated at least 3 times. To monitor YAP1 nuclear 371 translocation by western blot analysis, nuclear fractionation was performed using protein lysis 372 buffer containing 10mM HEPES, $1 \mathrm{mM} \mathrm{KCl,} \mathrm{1.5mM} \mathrm{MgCl2} \mathrm{and} \mathrm{10 \%} \mathrm{glycerol} \mathrm{(Buffer} \mathrm{A).} \mathrm{After}$ 373 washing the adherent cells with DPBS, samples were resuspended in Buffer A and incubated for 
3745 minutes on ice in the presence of $0.1 \%$ Triton-X-100. After centrifugation, the nuclear fraction

375 remained as a pellet while the supernatant corresponding to the cytosolic fraction. Nuclear

376 fraction was washed with Buffer A. After centrifugation, the nuclear fraction was resuspended in

377 RIPA buffer and samples were boiled for 5 min prior to sample loading. The cytosolic fraction

378 was centrifuged for $15 \mathrm{~min}$ at $13500 \mathrm{rpm}$. Only the supernatant was kept after centrifugation.

379 Western blot was performed as described before and the following antibodies were used for

380 protein detection: $\beta$-tubulin (Cell Signaling Technology, $\beta$-Tubulin (9F3) Rabbit mAb \#2128.

381 1:1000), PARP (Cell Signaling Technology, Rabbit mAb \#9542. 1:1000), YAP1 (Cell Signaling

382 Technology, YAP (D8H1X) XP® Rabbit mAb \#14074. 1:1000). For immunofluorescence in

383 adherent cells, samples were fixed with 4\% paraformaldehyde (Affymetrix; \#19943) for 7 min at

384 room temperature and permeabilized with iced-cold methanol. After blocking with 10\%

385 FBS:0.03\%Triton-X100, primary antibodies were incubated overnight in blocking solution ( $\beta$ -

386 actin and YAP1 antibodies detailed above). After three washes in DPBS:0.03\% Triton X-100)

387 cells were incubated with secondary antibodies for 45 minutes at room temperature [(Goat anti-

388 Mouse IgG $(\mathrm{H}+\mathrm{L})$ Highly Cross-Adsorbed Secondary Antibody, Alexa Fluor 594. Goat anti-

389 Rabbit IgG (H+L) Highly Cross-Adsorbed Secondary Antibody, Alexa Fluor 488)]. Cells were

390 rinsed with PBS-0.03\%-Triton three times and coverslips were mounted with ProLong Gold

391 antifade reagent with DAPI (\#P36935; Thermo Fisher Scientific). Images were captured using a

392 Leica DM IL microscope and registered using LAS software. For fluorescence intensity

393 quantification ImageJ software (National Institutes of Health, Bethesda, MD, USA) was used,

394 and statistical analyses were performed with GraphPad Prism software. 


\section{Immunohistochemistry and quantification}

FFPE tissue microarrays (ME1004h: Malignant melanoma, metastatic malignant melanoma and nevus tissue array) were obtained from US Biomax, Inc. (Derwood, MD). For the staining with anti-ZIP9 antibody [(SLC39A9 Antibody (PA5-52485), Thermofisher Scientific, Waltham, MA)], slides were deparaffinized and rehydrated following the standard immunohistochemistry protocol [(xylenes 5 minutes x 3, 100\% alcohol (5 min. x 3), 95\% alcohol (5 min.), 80\% alcohol (5 min.), 70\% alcohol (5 min.), and 50\% alcohol (5 min.) and finished with distilled water)]. The antigen retrieval was done by loading the slides into a retriever (Electron Microscopy Sciences EMS) with R-Buffer A. After 20 minutes, samples were allowed to cool for 30 minutes inside the retriever and for 20 minutes at room temperature. Samples were washed twice with PBS and blocked with Dako Dual Endogenous Enzyme Block (Code S2003. Agilent Santa Clara, CA) for 20 minutes at R.T. Samples were washed twice with PBS and blocked for 20 minutes with 2 drops of Vector Avidin Block. After washing twice with PBS, slides were blocked for 20 minutes with two drops of Vector Biotin Block (Avidin/Biotin Blocking kit. SP-2001. Vector

411 Laboratories, Inc. Burlingame, CA). Samples were washed twice with PBS with Protein Block

412 Serum-Free Ready-To-Use for 30 minutes at R.T. (Code X0909. Agilent Santa Clara, CA).

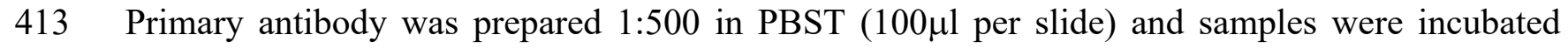
414 overnight at $4^{\circ} \mathrm{C}$. Samples were washed three times with PBS and incubated with Biotinylated 415 Secondary antibody (Vectastain Kit, Peroxidase Rabbit IgG, PK-4001) for one hour at R.T. After 416 three washes with PBS, ABC reagent (prepared 30 minutes in advance) was added and samples 417 were incubated for 30 minutes at R.T. Samples were washed twice with PBS and incubated for 3 418 minutes with ImmPACT® DAB Substrate, Peroxidase (HRP) (SK-4105. Vector laboratories, 419 Burlingame, CA). Tissues were counterstained with hematoxylin (30 seconds, R.T.) (GHS316. 
Sigma-Aldrich) dehydrated, and mounted with SecureMount (Fisher HealthCare ${ }^{\mathrm{TM}}$

421 PROTOCOL ${ }^{\text {TM }}$ Mounting Media. \#022-208. Fisher Scientific. Thermofisher Scientific). John T.

422 Seykora M.D., Ph.D, performed scoring of the stained tissue microarray, and scoring index was

423 determined by scoring the percentage of positive cells on a scale of 0 to 3 as well as the intensity

424 of ZIP9 staining on a scale of 0 to $4(1=1-25 \%, 2=26-50 \%, 3=51-75 \%, 4=76-100 \%)$.

425 The staining of the tissue microarrays (ME1004h) for AR detection was performed by 426 University of Pennsylvania Pathology Clinical Service Center-Anatomic Pathology Division,

427 using the highest grade, CLIA (Clinical Laboratory Improvement Amendments) certified and 428 validated test available. Briefly, five-micron sections of formalin-fixed paraffin-embedded tissue 429 were stained using antibody against Androgen Receptor [(Leica AR-318-L-CE, clone AR27 430 (clone AR27, 1:25)]. Staining was done on a Leica Bond-IIITM instrument using the Bond 431 Polymer Refine Detection System (Leica Microsystems DS9800). Heat-induced epitope retrieval 432 was done for 20 minutes with ER2 solution (Leica Microsystems AR9640). All the experiment 433 was done at room temperature. Slides are washed three times between each step with bond wash 434 buffer or water. The slides were reviewed and scored in blinded fashion by a board-certified U. 435 Penn pathologist. Prostate tissue was used as the positive control.

437 Immunocytochemistry

438 To detect ZIP9 protein in WM46 isogenic clones, the protocol described in ProSci $\Psi$ TM for 439 immunocytochemistry. Briefly, cells were fixed in 4\% PFA for 7 minutes. After two washes with 440 PBS (5min), cells were permeabilized with PBS/0.1\% Triton X-100 for 1 minute at R.T. Cells 441 were washed twice with PBS on a shaker. Once treated with $1.5 \% \mathrm{H}_{2} \mathrm{O}_{2} / \mathrm{PBS}$ solution for 15 442 minutes (R.T.), cells were washed again and blocked with 5\%BSA for one hour at R.T. For 
443 primary antibody incubation, $\alpha$-ZIP9 antibody [(SLC39A9 Antibody (PA5-52485),

444 Thermofisher Scientific, Waltham, MA)] was diluted 1:500 in 1\% BSA and cells were incubated

445 overnight at $4^{\circ} \mathrm{C}$. After washing three times with PBS on a shaker, the slide was incubated with

446 Biotinylated Secondary antibody (Vectastain Kit, Peroxidase Rabbit IgG, PK-4001) for one hour

447 at R.T. Cells were washed three times with PBS, ABC reagent (prepared 30 minutes in advance)

448 was added and samples were incubated for 30 minutes at R.T. After three washes with PBS,

449 samples were incubated for 1.5 minutes with Vector Laboratories DAB Peroxidase (HRP)

450 Substrate Kit (NC9276270. Vector laboratories, Burlingame, CA). Cells were counterstain with

451 hematoxylin (10 seconds, R.T.) (GHS316. Sigma-Aldrich) and mounted with SecureMount

452 (Fisher HealthCare ${ }^{\mathrm{TM}}$ PROTOCOL ${ }^{\mathrm{TM}}$ Mounting Media. \#022-208. Fisher Scientific.

453 Thermofisher Scientific).

\section{Cell membrane labeling with testosterone-BSA-FITC}

456 WM46 were seeded over coverslips in p12-well culture plate, after 24 hours cells TU2\% media

457 was removed, and cells were grown in Serum-free TU media for 24 hours. Cells were treated

458 with APA $(8 \mathrm{mM})$ or DMSO as vehicle control. After one hour, cells were treated with $0.25 \mu \mathrm{M}$

459 testosterone 3-(O-carboxymethyl) oxime:BSA-fluorescein isothiocyanate (T-BSA-FITC)

460 conjugate (T5771, Sigma-Aldrich, Munich, Germany) diluted in Tris-Buffer (pH 7.2) for 20 min

461 at room temperature. Negative control cells were incubated with $0.25 \mu \mathrm{M}$ BSA-FITC (A9771,

462 Sigma-Aldrich, Munich, Germany) diluted in Tris-Buffer ( $\mathrm{pH}$ 7.2) for 20 min at room

463 temperature. The medium was then aspirated, and cells were fixed for 7 minutes with $4 \%$ PFA.

464 Cells were washed three times with PBS (5 minutes each) and mounted with ProLong Gold 
465 antifade reagent with DAPI (\#P36935; Thermo Fisher Scientific). Images were captured using a

466 Leica DM IL microscope and registered using LAS software.

\section{Quantitative RT-PCR}

469 RNA was extracted using RNeasy kit (Qiagen. \#74104) following the manufacturer's 470 instructions. cDNA was obtained using High Capacity cDNA Reverse Transcription Kit 471 (Applied Biosystems \#4368814). For quantitative real-time PCR, PowerUP ${ }^{\mathrm{TM}} \mathrm{SYBR}^{\mathrm{TM}} \mathrm{Green}$ 472 Master Mix (Applied Biosystems \#A25741) was used. ViiA 7 Real-Time PCR System was used 473 to perform the reaction (Applied Biosystems). Values were corrected by $\beta$-actin expression. The $4742^{-\Delta \Delta \mathrm{Ct}}$ method was applied to calculate the relative gene expression. Primers used for the 475 amplification are included in Table S2.

Mice, subcutaneous tumors and pharmacologic treatments

478 All mice were purchased from Taconic Biosciences, Inc. (Rensselaer, NY, USA). 8- to 10-week 479 old male and female C57BL/6NTac or IcrTac:ICR-Prkdcscid mice were allowed to reach 480 sexually mature ages or to acclimatize for one week prior to being used in experiments. These 481 studies were performed without inclusion/exclusion criteria or blinding but included 482 randomization. Based on a two-fold anticipated effect, we performed experiments with at least 5 483 biological replicates. All procedures were performed in accordance with International Animal 484 Care and Use Committee (IACUC)-approved protocols at the University of Pennsylvania. 485 Subcutaneous tumors were initiated by injecting tumor cells in 50\% Matrigel (Corning, Bedford, 486 MA, USA) into the subcutaneous space on the right flanks of mice. $10 \times 10^{6}$ human WM46 or 10 487 x $10^{5}$ murine YUMM1.7 cells were used for each tumor. Oral administration of vehicle, 
488 bicalutamide $(30 \mathrm{mg} / \mathrm{kg} /$ day $)$ or apalutamide $(20 \mathrm{mg} / \mathrm{kg} /$ day $)$ was performed daily. $100 \mu \mathrm{l}$ of drug 489 was administrated by oral gavage [10\%DMSO:90\%Vehicle (15\% ethanol:85\% sesame oil)].

490 Weight and health status of the mice as well as tumor growth were monitored daily. As 491 subcutaneous tumors grew in mice, perpendicular tumor diameters were measured using calipers.

492 Volume was calculated using the formula $\mathrm{L} \times \mathrm{W}^{2} \times 0.52$, where $\mathrm{L}$ is the longest dimension and $493 \mathrm{~W}$ is the perpendicular dimension. Animals were euthanized when tumors exceeded a protocol494 specified size of $15 \mathrm{~mm}$ in the longest dimension. Secondary endpoints include severe ulceration, 495 death, and any other condition that falls within the IACUC guidelines for Rodent Tumor and 496 Cancer Models at the University of Pennsylvania.

\section{Statistical analysis}

499 For experiments comparing two groups (treated vs control), significance was calculated using the 500 Mann-Whitney test. For experiments with more than two groups, one-way ANOVA with 501 Tukey's honest significance difference test was performed. For the tumor growth studies in vivo, 502 non-linear regression analysis was performed to get the Exponential Growth Equation and 503 doubling times for tumor growth. To analyze the slopes of the curves and to compare them 504 between groups, linear regression analysis was performed. All the statistical analyses in this 505 work were performed using Graphpad Prism Software. Error bars represent standard error of the 506 mean $(\mathrm{SEM}) . * * * * \mathrm{p}$ value $\leq 0.0001 ; * * * \mathrm{p}$ value $\leq 0.001 ; * * \mathrm{p}$ value $\leq 0.01 ; * \mathrm{p}$ value $\leq 0.05$ n.s $>0.05$. 


\section{Acknowledgements}

512 The authors thank the University of Pennsylvania Skin Biology and Disease Research-based 513 center for analysis of tissue sections and University of Pennsylvania Pathology Clinical Service 514 Center-Anatomic Pathology Division for the AR staining of the tissue microarray. Funding:

515 T.W.R. is supported by grant from the NIH/NCI (R01 CA163566, R41CA228695), and by the

516 Stiefel award from the Dermatology Foundation. This work was also supported in part by the

517 Penn Skin Biology and Diseases Resource-based Center (P30-AR069589), the Melanoma 518 Research Foundation to T.W.R., and R37 GM56328 to J.K.F.

520 Author Contributions

521 C.A.P., R.P., A.T. performed the experiments. C.A.P., R.P. and J.K.F designed and performed 522 the experiments to study zinc influx. J.K.F provided experimental material and reviewed the 523 manuscript. J.T.S, scored the tissue microarray and reviewed the manuscript. C.A.P, C.A.N. and 524 T.W.R designed experiments and wrote the article. Competing interests: C.A.P., C.A.N., and 525 T.W.R., and are inventors on a provisional patent held by the University of Pennsylvania related 526 to this work.

\section{Conflict of interest}

529 T.W.R., C.A.N and C.A.P. are inventors on a provisional patent held by the University of 530 Pennsylvania related to this work. 


\section{The Paper Explained}

535 The Problem: Melanoma incidence and mortality are higher in males than females. This sex 536 difference remains, even after controlling for known melanoma risk factors such as

537 environmental and occupational exposures. While modern immune and targeted therapeutics

538 have markedly improved outcomes for many melanoma patients, most with metastatic tumors

539 still die from their disease. There is acute need for identification of new therapeutic targets, and

540 effective agents to safely engage them.

541 Results: Here, we define a nonclassical testosterone signaling pathway that establishes a novel 542 mechanistic link between male androgens and melanoma pathobiology. Using medically relevant 543 preclinical in vivo models, we first demonstrate that melanomas progress more rapidly in males

544 vs. females. We then show that $97 \%$ of primary melanomas and $100 \%$ of metastatic lesions from 545 people express the nonclassical membrane androgen receptor and zinc transporter, ZIP9, and that 546 ZIP9 activation promotes melanoma proliferation. Using complementary pharmacologic and 547 genetic approaches, we show that ZIP9 is a major determinant of the sex dependent difference in 548 tumor growth in vivo, and that ZIP9 may be an eminently druggable new therapeutic target for 549 melanoma.

550 Impact: ZIP9 is a zinc transporter that is not intentionally targeted by any available therapeutics 551 but is widely expressed in nearly all tissues. The demonstration that this receptor is 552 pharmacologically accessible suggests that inhibiting ZIP9 by repurposing currently approved 553 inhibitors designed to target the classical androgen receptor (AR) may be useful alone or in 554 combination with current standard of care therapeutics for a wide range of cancers, especially 555 those that disproportionately affect males. 


\section{$\underline{\text { References }}$}

560 1. Siegel, R. L., Miller, K. D. \& Jemal, A. Cancer statistics, 2020. CA Cancer J Clin 70, 7$56130(2020)$.

$562 \quad 2 . \quad$ GLOBOCAN2018. https://bit.ly/2Vd3C6a.

563 3. Clemmesen, J. \& Busk, T. Cancer Mortality Among Males and Females in Denmark,

564 England, and Switzerland: V. Incidence of Accessible and Inaccessible Cancers in Danish Towns and Rural Areas. Cancer Res 9, 415-421 (1949).

566 4. Hieken, T. J. et al. Sex-Based Differences in Melanoma Survival in a Contemporary

567 Patient Cohort. Journal of Women's Health (2020) doi:10.1089/jwh.2019.7851.

568 5. GLOBOCAN2018. Sex disparities in melanoma survival over time. shorturl.at/bkAI6.

569 6. Natale, C. A. et al. Activation of G protein-coupled estrogen receptor signaling inhibits melanoma and improves response to immune checkpoint blockade. Elife 7, (2018).

7. Natale, C. A. et al. Sex steroids regulate skin pigmentation through nonclassical membrane-bound receptors. Elife 5, (2016).

8. Sperling, M. A. Pediatric Endocrinology-Mean Sex Steriod Concentration in Infants, Children and adults. (Elsevier Health Sciences, 2014).

9. Barbosa-Desongles, A., Hernández, C., Simó, R. \& Selva, D. M. Testosterone induces cell proliferation and cell cycle gene overexpression in human visceral preadipocytes. Am. J. Physiol., Cell Physiol. 305, C355-359 (2013). differentiation via $\mathrm{G}$ protein-coupled receptors in the rat L6 skeletal muscle myoblast cell line. $J$. Cell. Physiol. 227, 98-107 (2012).

581 11. Rodríguez-Lozano, D. C., Piña-Medina, A. G., Hansberg-Pastor, V., Bello-Alvarez, C. \& 582 Camacho-Arroyo, I. Testosterone Promotes Glioblastoma Cell Proliferation, Migration, and Invasion Through Androgen Receptor Activation. Front. Endocrinol. 10, (2019).

584 12. Maasberg, M. et al. Androgen receptors, androgen-dependent proliferation, and 5 alphareductase activity of small-cell lung cancer cell lines. Int. J. Cancer 43, 685-691 (1989). 13. Allil, P. A. A., Visconti, M. A. \& Isoldi, A. M. L. C. and M. C. Photoperiod and

587 Testosterone Modulate Growth and Melanogenesis of S91 Murine Melanoma. Medicinal 588 Chemistry http://www.eurekaselect.com/82341/article (2008).

589 14. Meeth, K., Wang, J. X., Micevic, G., Damsky, W. \& Bosenberg, M. W. The YUMM 590 lines: a series of congenic mouse melanoma cell lines with defined genetic alterations. Pigment 591 Cell Melanoma Res 29, 590-597 (2016).

592 15. Gao, W., Bohl, C. E. \& Dalton, J. T. Chemistry and Structural Biology of Androgen 593 Receptor. Chem Rev 105, 3352-3370 (2005).

594 16. Askew, E. B., Gampe, R. T., Stanley, T. B., Faggart, J. L. \& Wilson, E. M. Modulation of 595 Androgen Receptor Activation Function 2 by Testosterone and Dihydrotestosterone. J. Biol.

596 Chem. 282, 25801-25816 (2007).

597 17. Berg, A. H., Rice, C. D., Rahman, M. S., Dong, J. \& Thomas, P. Identification and 598 characterization of membrane androgen receptors in the ZIP9 zinc transporter subfamily: I. 599 Discovery in female atlantic croaker and evidence ZIP9 mediates testosterone-induced apoptosis 600 of ovarian follicle cells. Endocrinology 155, 4237-4249 (2014).

601 18. Matsuura, W. et al. SLC39A9 (ZIP9) regulates zinc homeostasis in the secretory 602 pathway: characterization of the ZIP subfamily I protein in vertebrate cells. Biosci. Biotechnol.

603 Biochem. 73, 1142-1148 (2009). 
604 19. Fatfat, M. et al. Copper chelation selectively kills colon cancer cells through redox 605 cycling and generation of reactive oxygen species. BMC Cancer 14, 527 (2014).

606 20. He, L. et al. ZIP8, member of the solute-carrier-39 (SLC39) metal-transporter family: 607 characterization of transporter properties. Mol. Pharmacol. 70, 171-180 (2006).

608 21. Leal, M. F. et al. Clinical implication of 14-3-3 epsilon expression in gastric cancer.

609 World J. Gastroenterol. 18, 1531-1537 (2012).

610 22. Ruas, M. \& Peters, G. The p16INK4a/CDKN2A tumor suppressor and its relatives.

611 Biochim. Biophys. Acta 1378, F115-177 (1998).

612 23. Germann, U. A. et al. Targeting the MAPK Signaling Pathway in Cancer: Promising

613 Preclinical Activity with the Novel Selective ERK1/2 Inhibitor BVD-523 (Ulixertinib). Mol.

614 Cancer Ther. 16, 2351-2363 (2017).

615 24. Han, Y. Analysis of the role of the Hippo pathway in cancer. Journal of Translational

616 Medicine 17, 116 (2019).

617 25. Zhao, B., Li, L., Tumaneng, K., Wang, C.-Y. \& Guan, K.-L. A coordinated

618 phosphorylation by Lats and CK1 regulates YAP stability through SCF $\beta$-TRCP. Genes Dev 24,

619 72-85 (2010).

620 26. Fujii, M. Exploration of a new drug that targets YAP. J. Biochem. 152, 209-211 (2012).

621 27. Bulldan, A., Malviya, V. N., Upmanyu, N., Konrad, L. \& Scheiner-Bobis, G.

622 Testosterone/bicalutamide antagonism at the predicted extracellular androgen binding site of

623 ZIP9. Biochim Biophys Acta Mol Cell Res 1864, 2402-2414 (2017).

624 28. Ponnusamy, S. et al. Orally-Bioavailable Androgen Receptor Degrader, A Potential

625 Next-Generation Therapeutic for Enzalutamide-Resistant Prostate Cancer. Clin. Cancer Res.

626 (2019) doi:10.1158/1078-0432.CCR-19-1458.

627 29. Chi, K. N. et al. Apalutamide for Metastatic, Castration-Sensitive Prostate Cancer. $N$.

628 Engl. J. Med. 381, 13-24 (2019).

629 30. Kayigil, O., Atahan, O. \& Metin, A. Cyproterone acetate monotherapy in advanced

630 prostatic carcinoma. Int Urol Nephrol 29, 213-220 (1997).

631 31. Fukada, T. \& Kambe, T. Molecular and genetic features of zinc transporters in

632 physiology and pathogenesis. Metallomics 3, 662-674 (2011).

633 32. Costello, L. C., Liu, Y., Zou, J. \& Franklin, R. B. Evidence for a zinc uptake transporter

634 in human prostate cancer cells which is regulated by prolactin and testosterone. J Biol Chem 274,

635 17499-17504 (1999).

636 33. Bafaro, E., Liu, Y., Xu, Y. \& Dempski, R. E. The emerging role of zinc transporters in

637 cellular homeostasis and cancer. Signal Transduction and Targeted Therapy 2, 1-12 (2017).

638 34. Ma, M. et al. Sustained androgen receptor signaling is a determinant of melanoma cell

639 growth potential and tumorigenesis. J Exp Med 218, (2021).

$64035 . \quad$ Klein, S. L. \& Flanagan, K. L. Sex differences in immune responses. Nat. Rev. Immunol.

641 16, 626-638 (2016).

642 


\section{Figure legends}

647 Fig. 1: Biologic sex and testosterone promote proliferation of melanoma models lacking

648 detectable AR. Tumor growth of human WM46 melanoma in male and female immunodeficient

649 SCID mice. Tumor doubling times are 16.2 and 18.6 days for males and females respectively

650 (Non-linear regression analysis/Exponential fit. See also Sup. Fig. 1 for expanded statistical

651 analysis). B. Cell proliferation (cell number) determined after 6 days of treatment with vehicle

652 (DMSO), 100nM testosterone (T) or 100nM dihydrotestosterone (DHT). Human primary

653 melanocytes (H.Mel.) and human melanoma WM46 cells are shown. Graphs represent the

654 average of three independent experiments. C. Cell proliferation of human WM46 and murine

655 YUMM1.7 melanoma cells exposed to increasing concentrations of testosterone (T) (left panels)

656 or dihydrotestosterone (DHT) (right panel). D. AR mRNA expression in primary melanocytes

657 (L: low melanin content; D: High melanin content), a battery of human melanoma lines, and

658 murine YUMM1.7 determined by qPCR. VCaP cell line (prostate cancer) was used as a positive

659 control. E. AR protein expression determined by Western blot with two different antibodies.

660 Upper line $=$ Androgen Receptor [(D6F11) XP® Rabbit mAb \#5153. Lower line $=$ Recombinant

661 Anti-Androgen Receptor antibody [EPR1535(2)] (ab133273). Two human prostate cancer lines

662 were used as a positive control (VCaP and LNCaP). $\beta$-actin was used as loading control.

$663 * * \mathrm{p}$ value $\leq 0.01 ; * \mathrm{p}$ value $\leq 0.05$

664

665 Fig 2: ZIP9 is active in human melanoma cells, and is broadly expressed in human

666 melanocytic tumors. A. ZIP9 protein expression determined by western blot in primary

667 melanocytes and a battery of human melanoma cell lines. B. Time-lapse in vivo analysis of $\mathrm{Zn}^{++}$

668 influx in WM46 cells upon testosterone addition (100nM). FluoZin-3 was used as $\mathrm{Zn}^{++}$reporter.

669 C. Intracellular levels of $\mathrm{Zn}^{++}$after long-term testosterone treatment (96 hours; 100nM 
670 testosterone). Zinc levels were measured as fluorescence intensity per cell. FluoZin-3 was used

671 as $\mathrm{Zn}^{++}$reporter. Representative images are shown on the right at indicated time-points. D.

672 WM46 relative proliferation (cell number) after 6 days in the presence of $100 \mathrm{nM}$ testosterone

673 (T) +/- $200 \mathrm{nM}$ zinc chelator (TPEN). E. WM46 proliferation in the presence exogenous divalent

674 cations $\mathrm{Zn}^{++} \mathrm{Fe}^{++}$and $\mathrm{Mn}^{++}$. Cells were grown for 6 days and treated as indicated in the legend.

675 Error bars represent standard error of the mean (SEM). F. Validation of ZIP9 [(SLC39A9

676 Antibody (PA5-52485)] and androgen receptor [(Leica AR-318-L-CE, clone AR27 (clone AR27,

677 1:25)] antibodies for immunohistochemistry. ZIP9 staining performed in wild-type and ZIP9

678 knock-out cells. Prostate gland tissue and human prostate cancer samples were used as positive

679 controls for AR. Representative images of human melanoma samples stained for ZIP9 and AR.

680 Tumors expressing low, medium and high levels of ZIP9 are shown. Replicates from the same

681 samples stained for AR are shown. 20X magnification (1.6X zoom). Scale bar=60 $\mu$ M. G.

682 Graphic representation of the \% of tumors that express ZIP9. Data from nevus, primary

683 melanomas and metastatic melanoma are displayed. H. Graphic representation of the percentage

684 of nevi, primary lesions and metastatic tumors classified according to ZIP9 intensity (Score 1=1$25 \%, 2=26-50 \%, 3=51-75 \%, 4=76-100 \%)$.

686

$* * * * \mathrm{p}$ value $\leq 0.0001 ; * * \mathrm{p}$ value $\leq 0.01 ; * \mathrm{p}$ value $\leq 0.05 ; \mathrm{n} . \mathrm{s}>0.05$.

687

688 Fig. 3: ZIP9 mediates testosterone effects in melanoma. A. Proliferation (cell number) of

689 isogenic clonal populations of WM46 wtZIP9 and $\triangle$ ZIP9 (gRNA \#3) cells exposed to increasing

690 concentrations of testosterone (T) and dihydrotestosterone (DHT). B. Proliferation (cell number)

691 of isogenic clonal populations of WM46 wtZIP9 and $\Delta$ ZIP9 (gRNA \#4) cells exposed to

692 increasing concentrations of testosterone (T) and dihydrotestosterone (DHT). The graph

693 represents the average of three independent experiments. C. Intracellular levels of zinc in human 
694 melanoma $\triangle$ ZIP9 cells measured as relative fluorescence intensity of FluoZin-3. Graphs

695 represent the average of three independent experiments. D. Relative cell proliferation in the 696 presence of $100 \mathrm{nM}$ testosterone (T), zinc chelator (200nM TPEN) and $\mathrm{ZnCl}_{2}(400 \mathrm{nM})$. Cells 697 were cultured for 6 days. The graph represents the average of three independent experiments. $* * * * \mathrm{p}$ value $\leq 0.0001 ; * * \mathrm{p}$ value $\leq 0.01 ; * \mathrm{p}$ value $\leq 0.05 ; \mathrm{n} . \mathrm{s}>0.05$

Fig. 4: Testosterone driven increase in melanoma proliferation requires ZIP9 and

activation of MAPK and YAP1. A. RPPA analysis displaying changes in protein expression in WM46 human melanoma cells following exposure to $100 \mathrm{nM}$ testosterone $(\mathrm{T})$ for increasing amounts of time. Down-regulated proteins are shown in green; red color corresponds to upregulated proteins and control proteins showing no fold-change when compared to vehicletreated cells are shown in blue. B. Relative proliferation (cell number) after exposure to pharmacologic ERK1/2 inhibition via 50nM RVD-523 (Ei) alone or in combination with testosterone. wtZIP9 and $\triangle$ ZIP9 WM46 cells are shown. The western blot shows levels of phosphorylation of the ERK target RSK in wtZIP9 and $\triangle$ ZIP9 WM46 cells (Ei represents RVD523). C. Western blot for YAP1 in fractionated WM46 lysates. Cells were treated with $100 \mathrm{nM}$ testosterone for the indicated times. $\beta$-Actin is used as cytoplasmic fraction positive control.

711 PARP is used as nuclear fraction positive control. D. Quantification of YAP-1 nuclear

712 immunodetection after 30 minutes of exposure to $100 \mathrm{nM}$ testosterone (T) and/or $8 \mu \mathrm{M}$

713 dobutamine (Dob) in wtZIP9 and $\triangle$ ZIP9 cells. E. Proliferation of wtZIP9 and $\triangle$ ZIP9 WM46

714 cells after treatment with 100nM testosterone (T) and/or the YAP inhibitor dobutamine (Dob). F.

715 Relative mRNA expression of YAP1 target genes after 30 minutes in the presence of $100 \mathrm{nM}$

716 testosterone and/or $8 \mu \mathrm{M}$ dobutamine. Error bars represent standard error of the mean (SEM). 
$* * * \mathrm{p}$ value $\leq 0.001 ; * * \mathrm{p}$ value $\leq 0.01 ; * \mathrm{p}$ value $\leq 0.05 ; \mathrm{n} . \mathrm{s}>0.05$.

Fig. 5: Pharmacologic ZIP9 blockade inhibits melanoma in vivo. A. Proliferation of human melanoma cells (WM46) in the presence of $100 \mathrm{nM}$ testosterone (T) $+/-2 \mu \mathrm{M}$ AR inhibitors (BIC:Bicalutamide; ENZ:Enzalutamide; APA:Apalutamide). Western blot showing ERK and pERK proteins in WM46 cells treated with 100nM testosterone $+/-2 \mu \mathrm{M}$ BIC $+/-200 \mathrm{nM}$ zinc

3, SK-MEL-2 and YUMM1.7) treated with $100 \mathrm{nM}$ testosterone (T) in combination with apalutamide $(2 \mu \mathrm{M})(\mathrm{APA})$. C. Cell membrane labeling with cell impermeable testosterone-BSA conjugated with FITC $(0.25 \mu \mathrm{M})$. BSA-FITC $(0.25 \mu \mathrm{M})$ was used as a negative control for unspecific binding. Quantification of membrane labeling with T-BSA or the control BSA. The graph represents the fluorescence intensity relative to the total area of each cell.

D. Proliferation of WM46 wtZIP9 and $\triangle$ ZIP9 treated with $100 \mathrm{nM}$ testosterone $(\mathrm{T})$ in combination with apalutamide $(2 \mu \mathrm{M})$ (APA). E. Tumor growth and survival analysis in SCID male mice bearing

731 WM46 derived subcutaneous tumors (APA treatment: $20 \mathrm{mg} / \mathrm{kg} /$ day via oral gavage). ** p732 value $<0.005$ by ANOVA. Doubling time (non-linear regression analysis): Vehicle=11.11 days;

$733 \mathrm{APA}=25.22$ days. F. Working model: ZIP9 activation promotes ERK phosphorylation and 734 induces YAP1 nuclear translocation. AR inhibitors [represented in the figure by apalutamide 735 (APA)] block testosterone effects through ZIP9 inactivation. $* * * * \mathrm{p}$ value $\leq 0.0001 ; * * * \mathrm{p}$ value $\leq 0.001 ; * * \mathrm{p}$ value $\leq 0.01 ; * \mathrm{p}$ value $\leq 0.05 ; \mathrm{n} . \mathrm{s}>0.05$ 


\section{Figures}

\section{Figure 1}

A

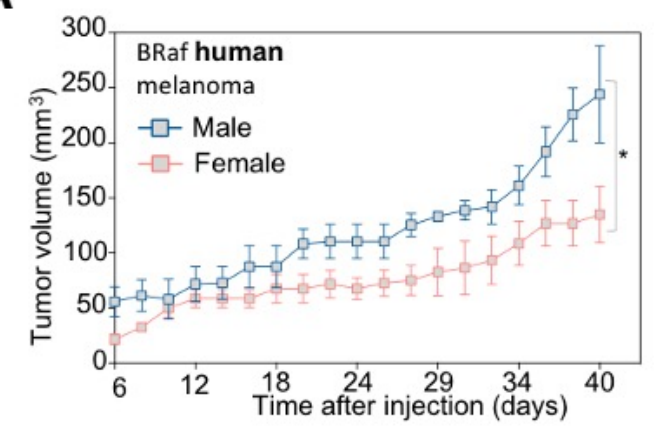

C
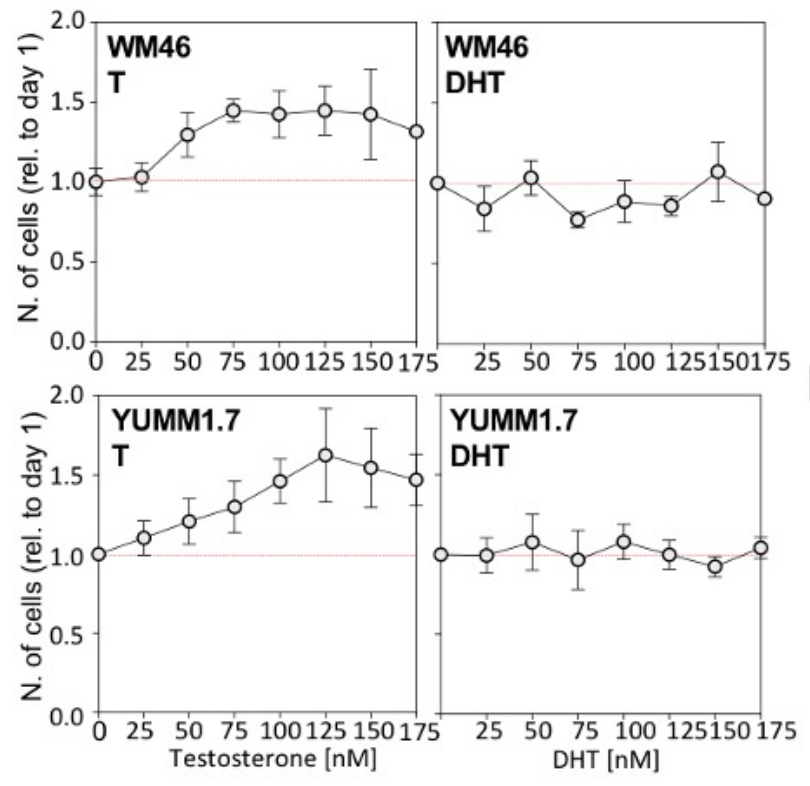

B

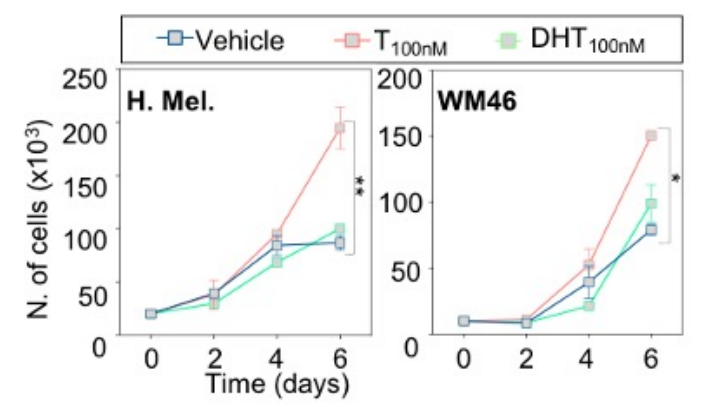

D

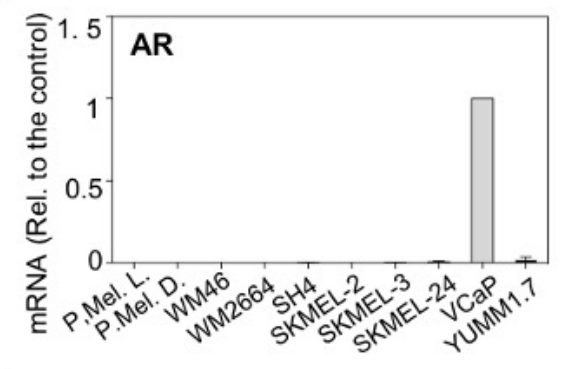

E

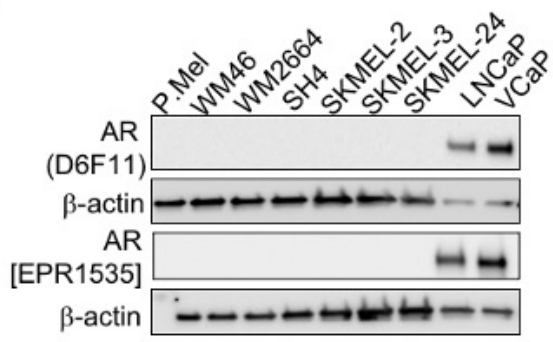

741 
bioRxiv preprint doi: https://doi.org/10.1101/2020.03.12.989160; this version posted March 3, 2021. The copyright holder for this preprint (which was not certified by peer review) is the author/funder. All rights reserved. No reuse allowed without permission.

\section{Figure 2}

A

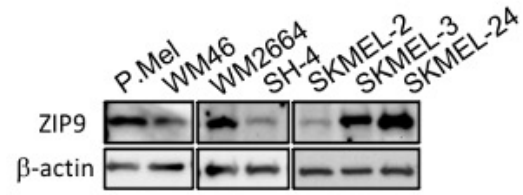

C

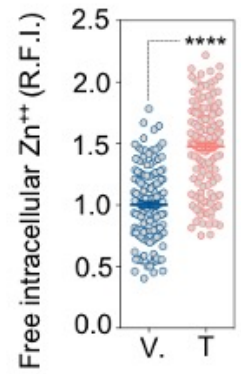

$\mathbf{F}$
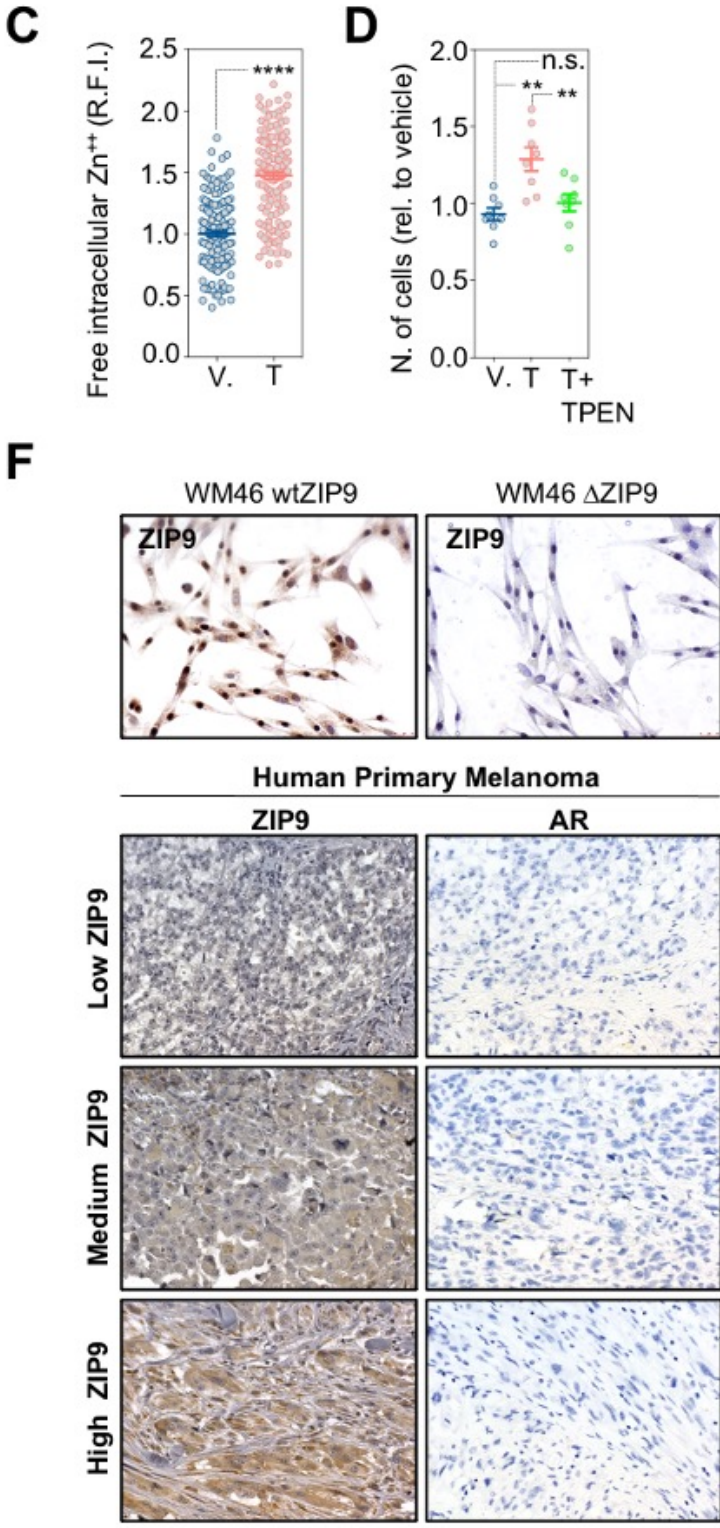

G

$\%$ of tumors expressing ZIP9

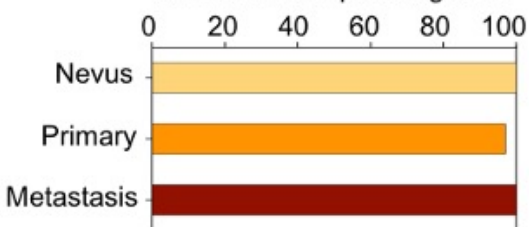

B

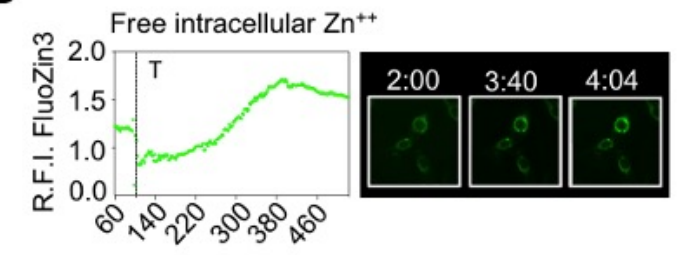

E

Time (Secs)

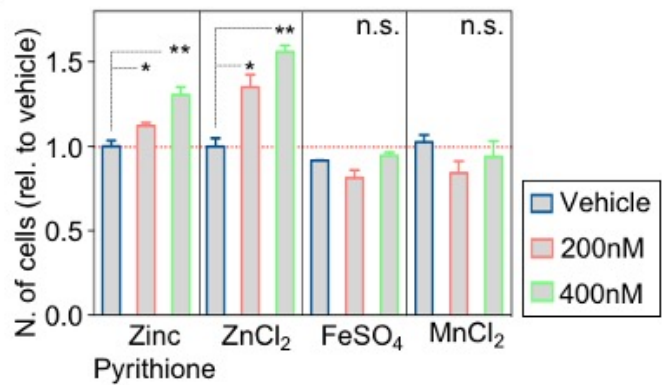

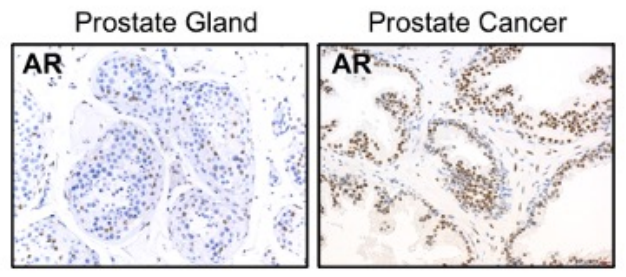

Human Metastatic Melanoma

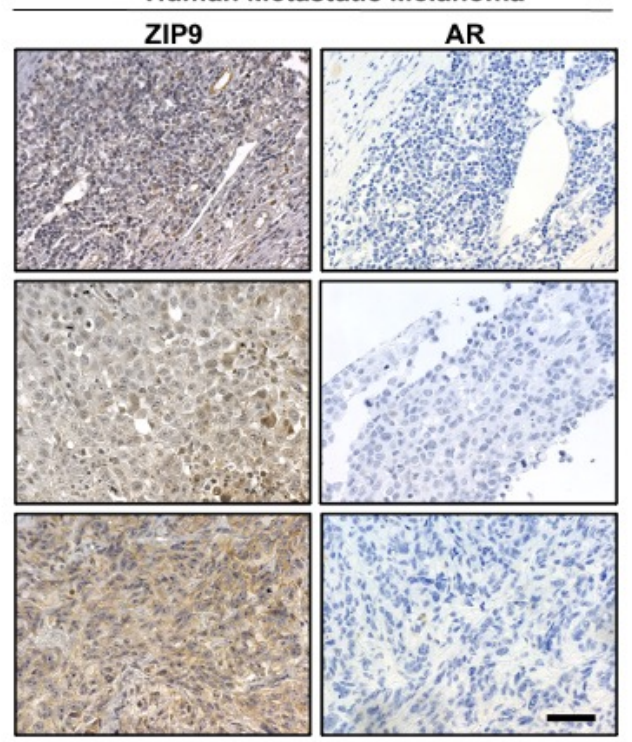

H

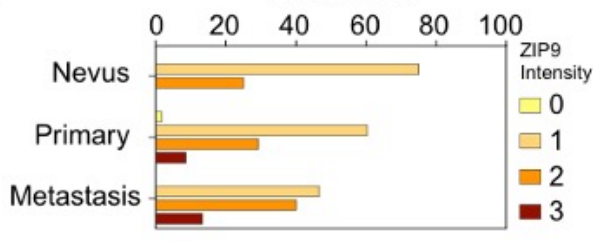




\section{Figure 3}

A
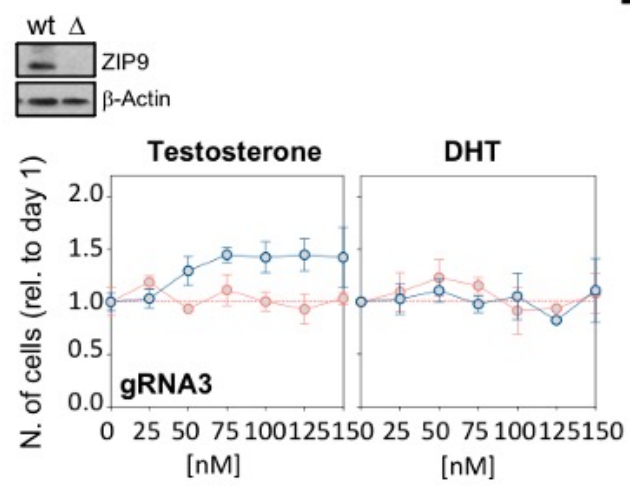

C

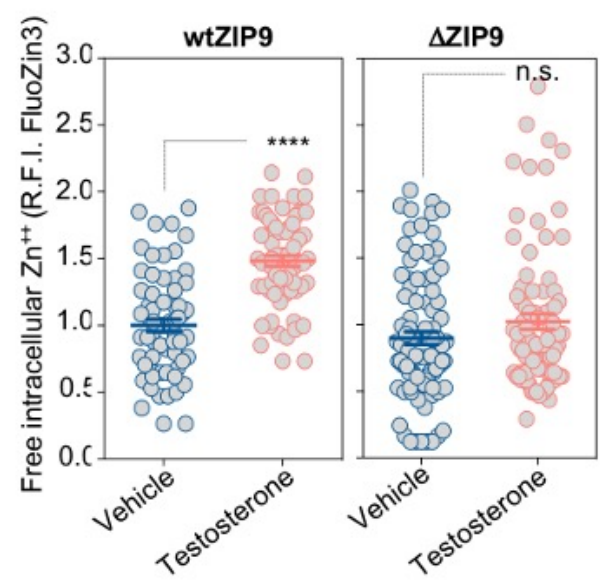

B

\begin{tabular}{|l|l} 
wt $\Delta$ & \\
- & ZIP9 \\
\hline$-\infty$ & $\beta$-Actin
\end{tabular}

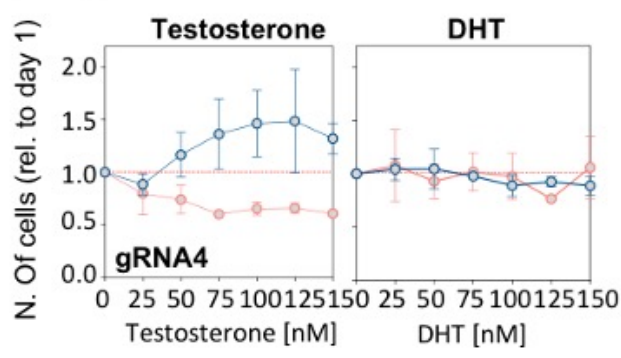

D

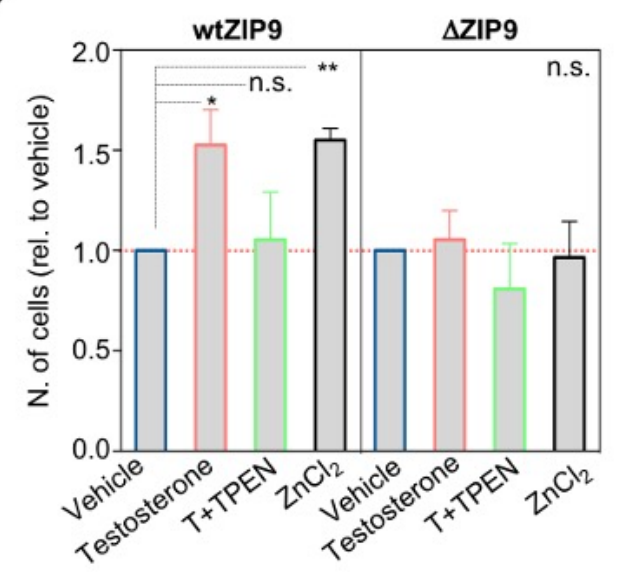




\section{Figure 4}

A

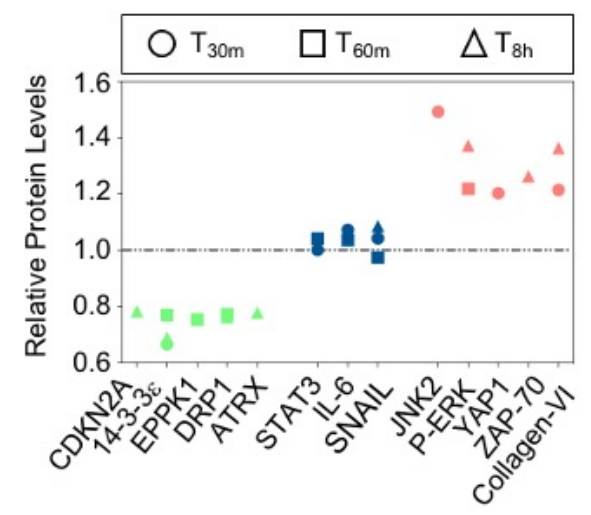

C

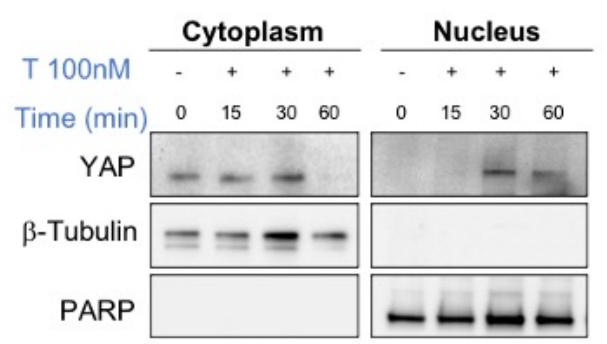

E

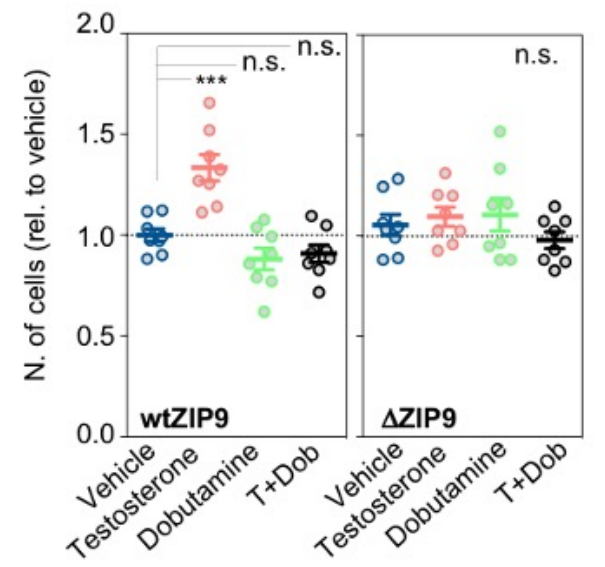

B

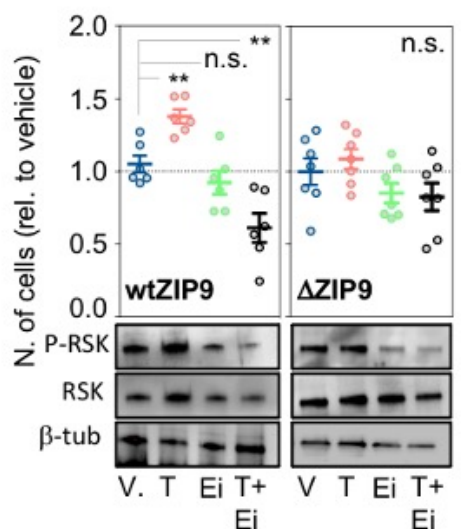

D

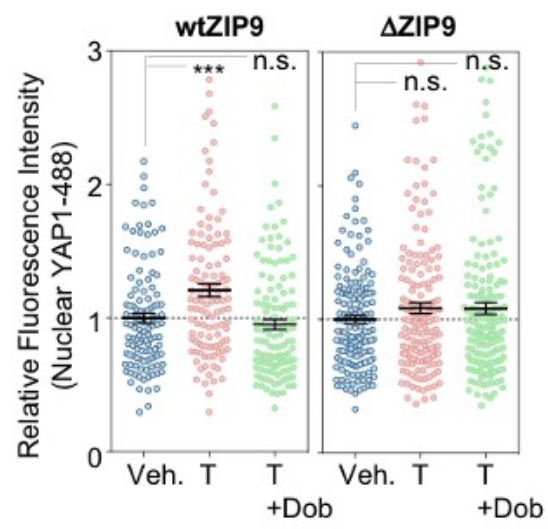

F

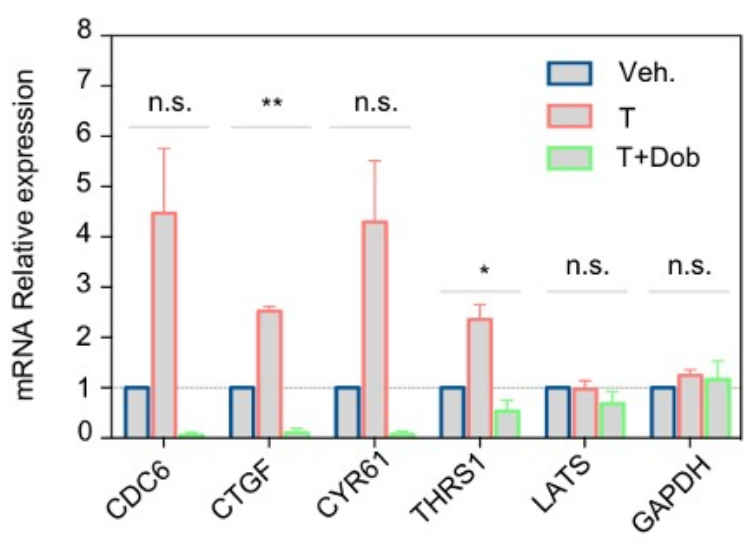




\section{Figure 5}

A

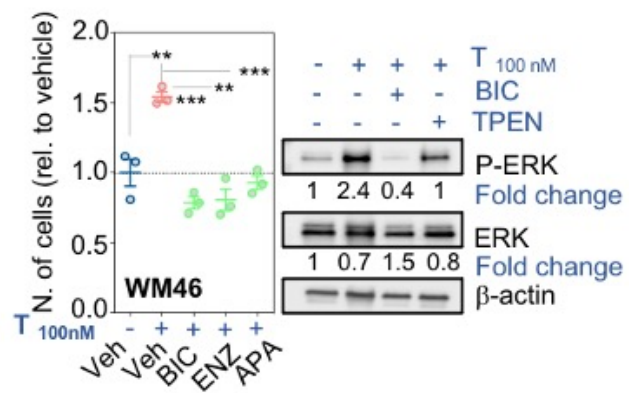

B

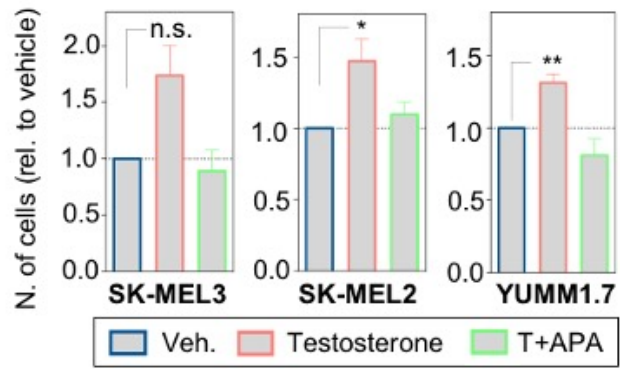

C
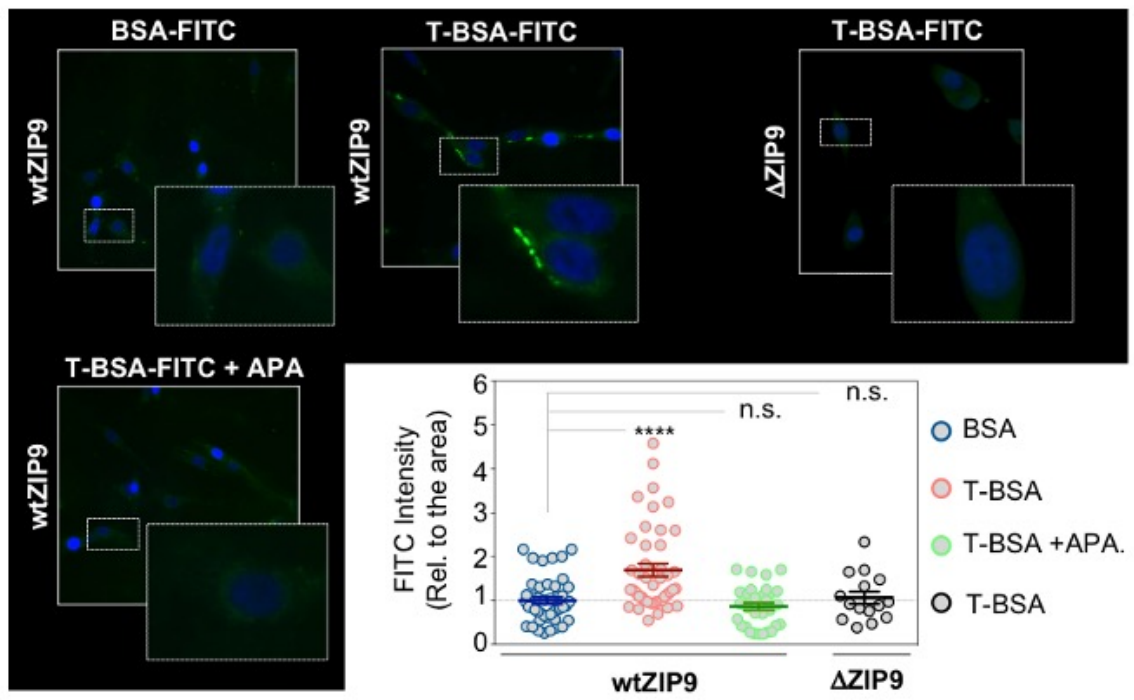

D

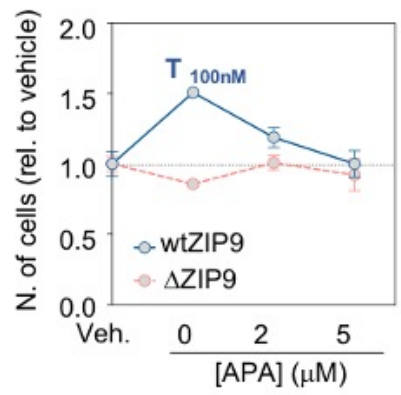

F

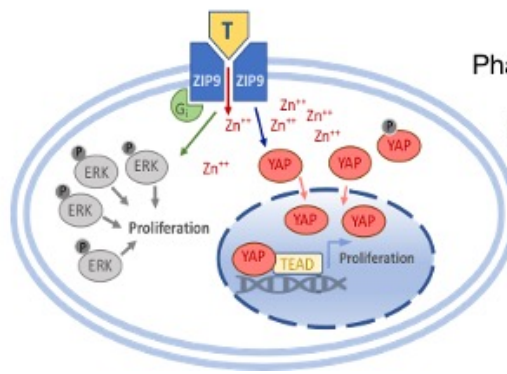

E
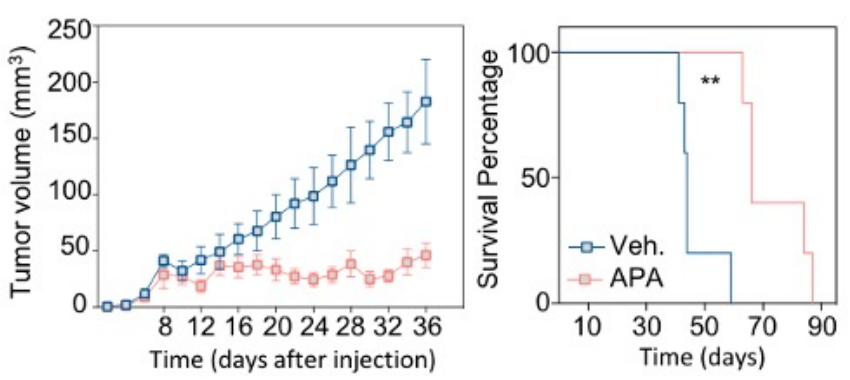


\section{Supplementary Figures}

\section{Supplementary Figure 1}

A

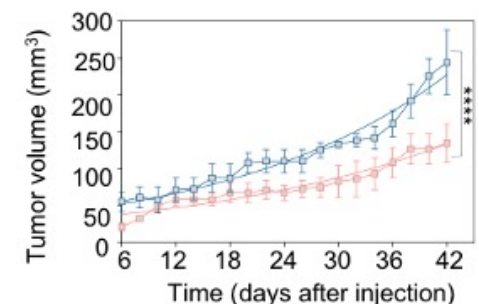

Time (days after injection)

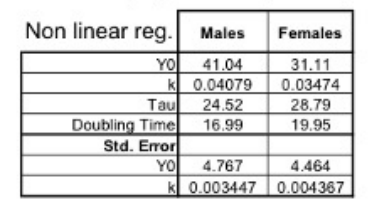

B

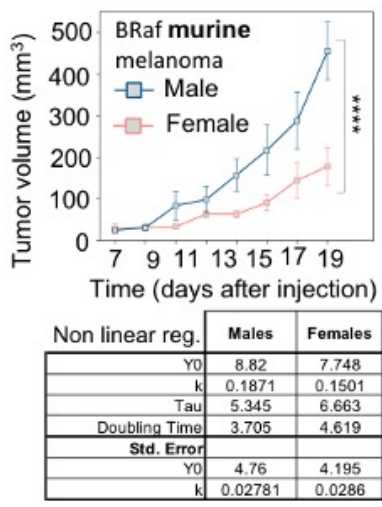

C

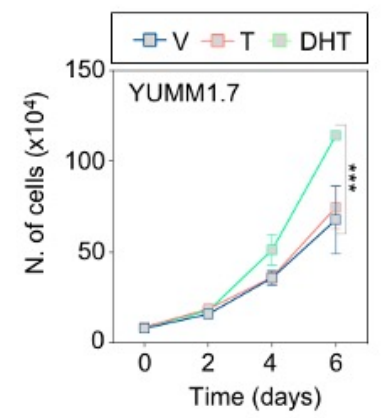

D

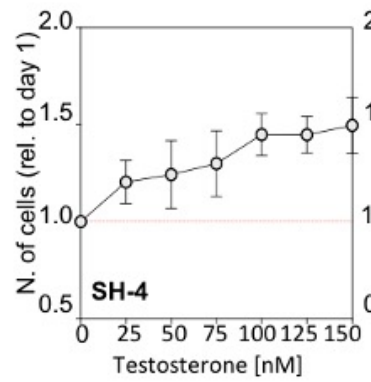

E

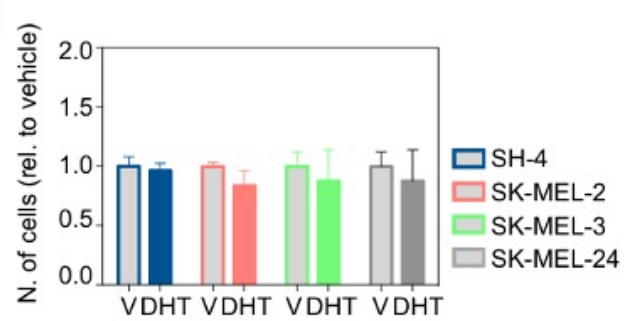

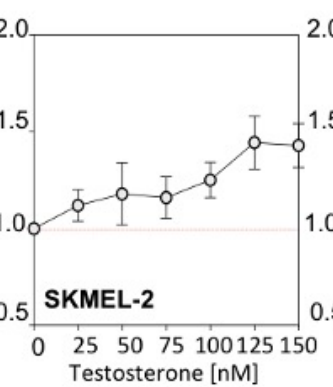

SKMEL-3

$25 \quad 5075100125150$ Testosterone $[\mathrm{nM}]$

$\mathbf{F}$

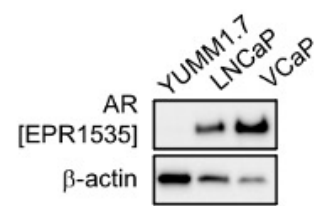

G

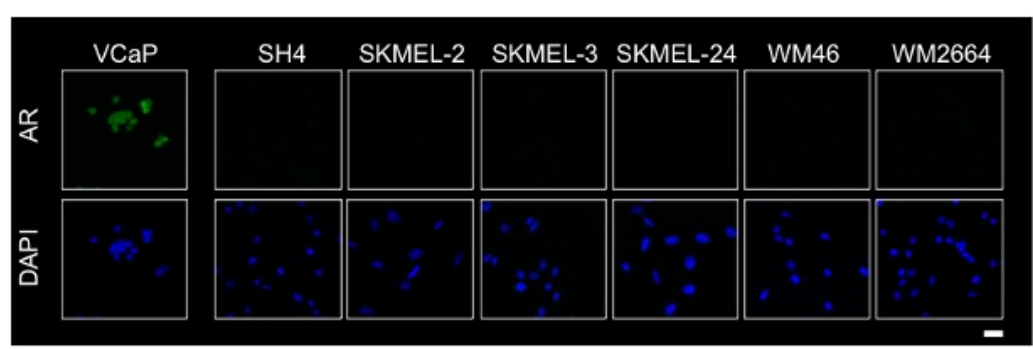


753 Fig. S1. Non-linear regression analysis (Exponential fit) for males and females. Doubling time

754 for tumor growth: males=16.99 days and females=19.95 days. (Linear-regression analysis for

755 slope comparison: $* * * *$ p-value $<0.0001)$. B. Tumor growth in C57BL/6 male and female mice

756 bearing syngeneic murine melanoma (YUMM1.7). Doubling time for tumor growth: males $=3.7$

757 days, females $=4.6$ days (Linear-regression analysis for slope comparison: $* * * *$ p-

758 value $<0.0001)$. C. YUMM1.7 cell number determined after 6 days of treatment with vehicle

759 (DMSO), $100 \mathrm{nM}$ testosterone (T) or $100 \mathrm{nM}$ dihydrotestosterone (DHT). Graphs represent the

760 average of three independent experiments. Error bars represent standard error of the mean

761 (SEM). D. Relative cell proliferation of human melanoma cell lines treated for 6 days with

762 increasing concentrations of testosterone (T). E. Relative cell proliferation of human melanoma

763 cells treated for 6 days with dihydrotestosterone (DHT $100 \mathrm{nM}$ ). F. AR protein expression

764 determined by Western blot in YUMM1.7 cells. G. Immunofluorescence detecting AR (D6F11)

765 in a battery of human-derived melanoma cells. AR is shown in green; DNA was stained with

766 DAPI and it is shown in blue. Scale bar $25 \mu \mathrm{M}$. Error bars represent standard error of the mean

767 (SEM). $* * * * \mathrm{p}$ value $\leq 0.0001 ; * * * \mathrm{p}$ value $\leq 0.001$

768

769

770

771

772

773

774

775 


\section{Supplementary Figure 2}
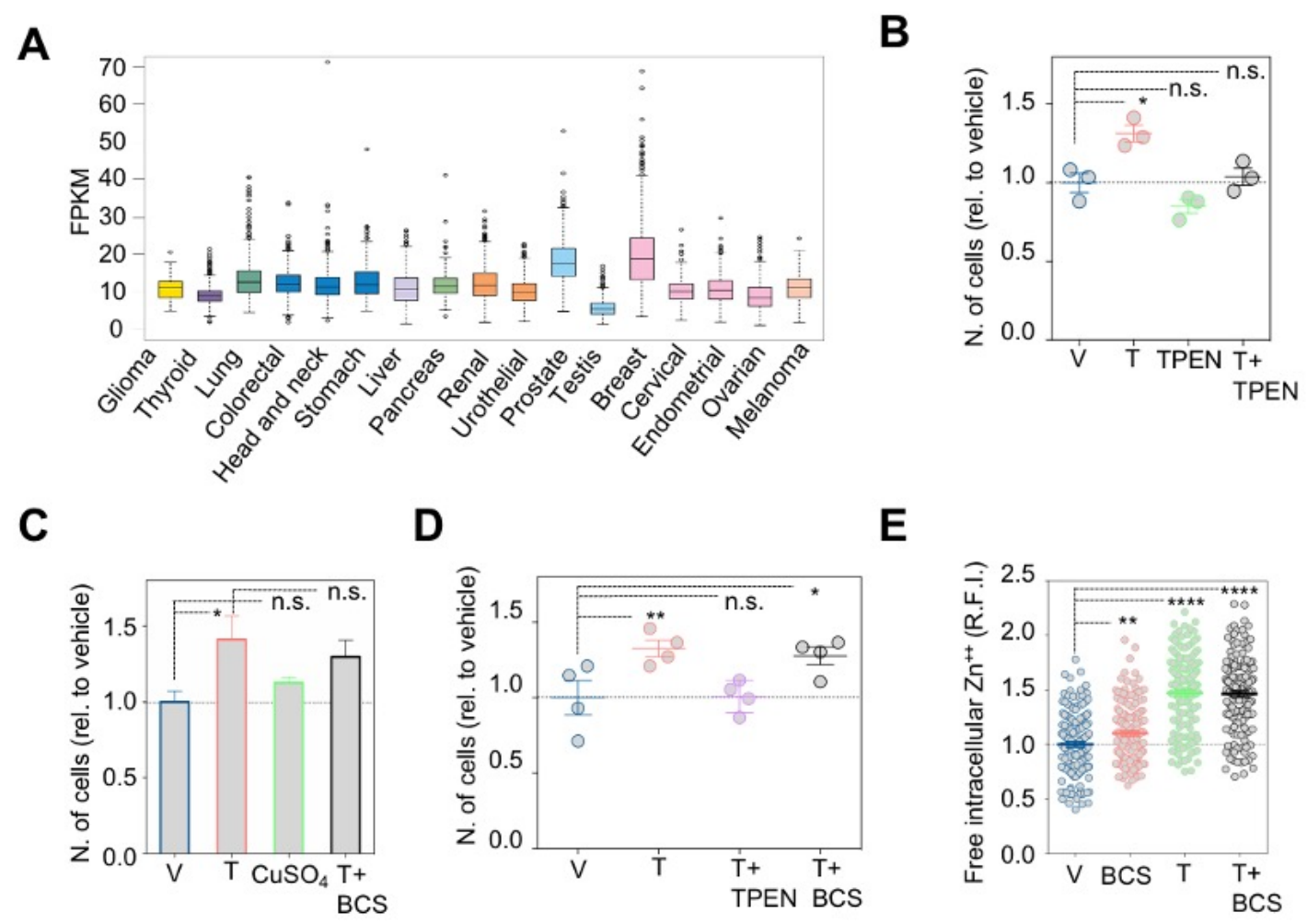

E

Fig. S2. A. RNA-seq data from 17 cancer types reported as median FPKM (number Fragments

Per Kilobase of exon per Million reads), generated by the The Cancer Genome Atlas (TCGA). RNA cancer tissue category calculated based on mRNA expression levels across all 17 cancer tissues and include: cancer tissue enriched, cancer group enriched, cancer tissue enhanced, expressed in all, mixed and not detected. Normal distribution across the dataset is visualized with

782 box plots, shown as median and 25 th and 75 th percentiles. Points are displayed as if they are

783 above or below 1.5 times the interquartile range. B. WM46 relative proliferation (cell number) in

784 presence of $100 \mathrm{nM}$ testosterone $(\mathrm{T})+/-200 \mathrm{nM}$ zinc chelator TPEN. C. WM46 proliferation

785 treated with $100 \mathrm{nM}$ testosterone $(\mathrm{T}),+/$ - copper sulfate $(\mathrm{CuSO} 4)$ and copper chelator $(25 \mu \mathrm{M}$

786 BCS). D. Relative cell proliferation of WM46 cells treated with testosterone $(100 \mathrm{nM})+/-$ zinc

787 chelator TPEN $(200 \mathrm{nM})$ and the copper chelator BCS $(25 \mu \mathrm{M})$. E. Relative intracellular $\mathrm{Zn}^{++}$in 
788 cells treated with testosterone and/or copper chelator (BCS). The graph represents relative

789 fluorescence intensity. Error bars represent standard error of the mean (SEM).

$790 * * * * \mathrm{p}$ value $\leq 0.0001 ; * * \mathrm{p}$ value $\leq 0.01 ; * \mathrm{p}$ value $\leq 0.05 ; \mathrm{n} . \mathrm{s}>0.05$

791

792

793

794

795

796

797

798

799

800

801

802

803

804

805

806

807

808

809

810 


\section{Supplementary Figure 3}

A

Fig. S3. Graphic representation of ZIP9 staining intensity in human samples from nevus, primary

814 melanoma and metastatic melanoma in males vs. females (Score $1=1-25 \%, 2=26-50 \%, 3=51$ -

$81575 \%, 4=76-100 \%)$. n.s $>0.05$.

816

817 


\section{Supplementary Figure 4}

A

\begin{tabular}{cccc} 
ICE Analysis_Synthego & Indel \% & Model Fit (R2) & $\begin{array}{c}\text { Knock-out } \\
\text { Score }\end{array}$ \\
\hline \hline WM46 wIZIP9 gRNA 3. Clone 3.3 & 0 & 1 & 0 \\
WM46 koZIP9 gRNA 3. Clone 3.2 & 92 & 0.92 & 92 \\
WM46 wIZIP9 gRNA 3. Clone 4.6 & 0 & 1 & 0 \\
WM46 koZIP9 gRNA 4. Clone 4.b & 90 & 0.92 & 90 \\
YUMM1.7 koZIP9 gRNA 3. Clone 3.3 & 90 & 0.9 & 90
\end{tabular}

C

YUMM1.7 koZIP9 Clone 3.2.9

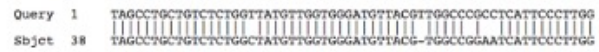

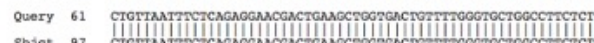

D

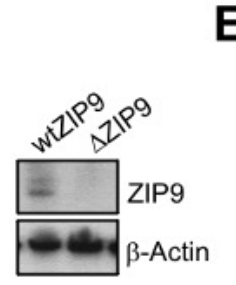

E

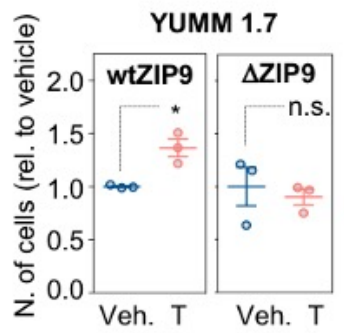

$\mathbf{F}$

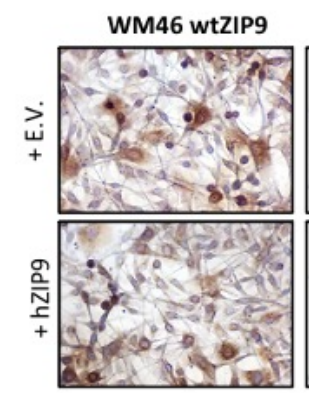

E.V. hZIP9

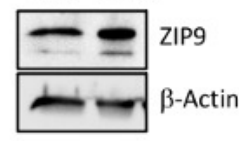

WM46 $\Delta$ ZIP9

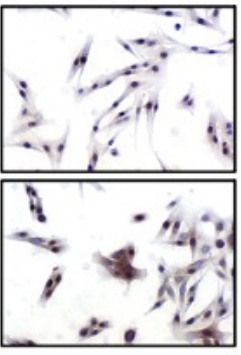

E.V. hZIP9

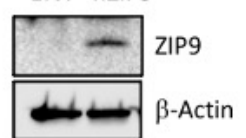

B

WM46 vs hZIP9 sequence (ENSMBL)

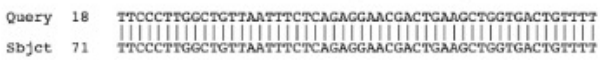

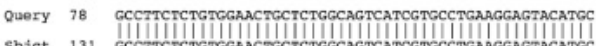

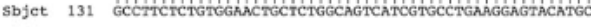

WM46 wtZIP9 Clone 4.6

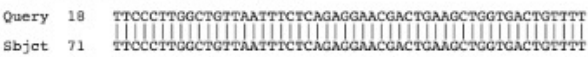

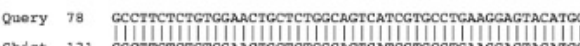

WM46 wtZIP9 Clone 3.3

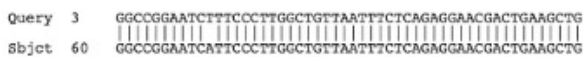

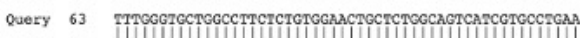

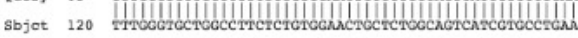

WM46 koZIP9 Clone 4.b

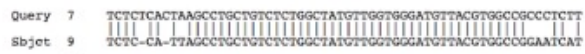

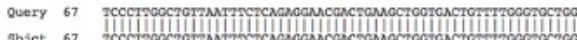

\section{WM46 koZIP9 Clone 3.2}

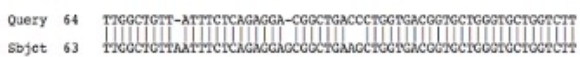

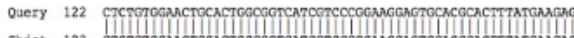

G

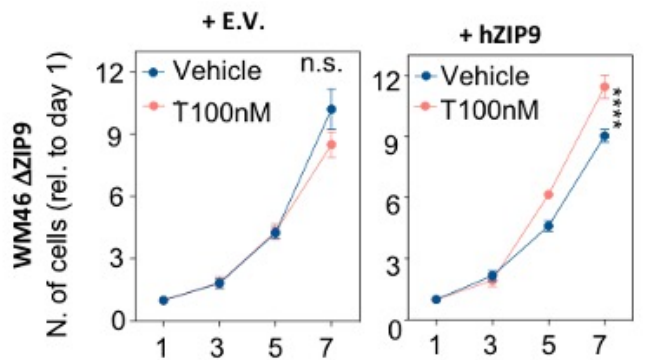

837 Fig. S4. A. Table shows the knock-out scores for WM46 isogenic clones obtained using ICE

838 Analysis software (Synthego). B. Blast alignment of the DNA sequences surrounding the gRNA

839 target sequence and SLC39A9 (Ensmbl. Homo Sapiens_SLC39A9_202) are shown for each 
841 with human SLC39A9 (Ensmbl. Homo Sapiens_SLC39A9_202). Query = SLC39A9_202; Sbjct

$842=$ isogenic clone sequences C. Target sequence of YUMM1.7 isogenic clone was aligned with

843 mus musculus Slc30a9 sequence. D. Western blot showing ZIP9 expression in wild type and

844 knock-out YUMM1.7 isogenic clones. E. Relative proliferation (cell number) of YUMM1.7

845 wtZIP9 and $\triangle$ ZIP9 in the presence of $100 \mathrm{nM}$ testosterone (T) for 6 days. The graphs represent

846 the average of three independent experiments. Error bars represent standard error of the mean

847 (SEM). * p value $\leq 0.05$. F. Immunocytochemistry in WM46 isogenic clones transduced with

848 control pRRL lentivector (E.V.) or hZIP9 expressing lentivector. Western blots below show the

849 proteins in each sample 72 hours after transduction. G. Relative cell proliferation (cell number)

850 with testosterone treatment $(100 \mathrm{nM})$ for 7 days in WM46 $\Delta$ ZIP9 isogenic clones transduced

851 with empty pRRL lentivector (E.V.) (left panel) or pRRL_hZIP9 expressing vector (right panel).

852 Error bars represent standard error of the mean (SEM). **** p value $\leq 0.0001 ; \mathrm{n} . \mathrm{s}>0.05$. 


\section{Supplementary Figure 5}

A

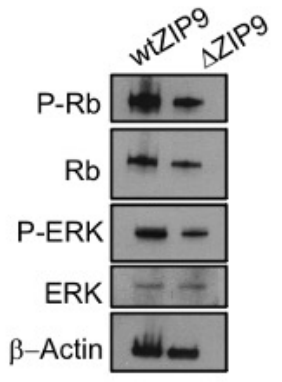

C

Restored expression of ZIP9 in WM46 $\triangle$ ZIP9

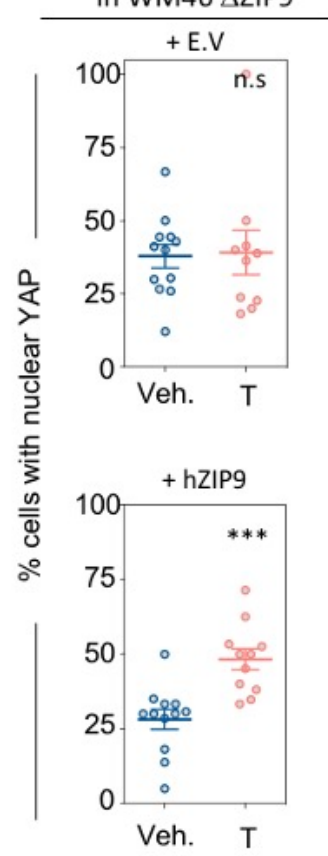

B

\begin{tabular}{lcc} 
WM46 wtZIP9 & \\
\hline Vehicle & Testosterone & Testosterone + \\
$\left(\mathrm{T}_{30 \mathrm{~min}}\right)$ & $\left(\mathrm{T}_{30 \mathrm{~min}}\right)$ & Dob $\left(\mathrm{T}_{30 \mathrm{~min}}\right)$
\end{tabular}

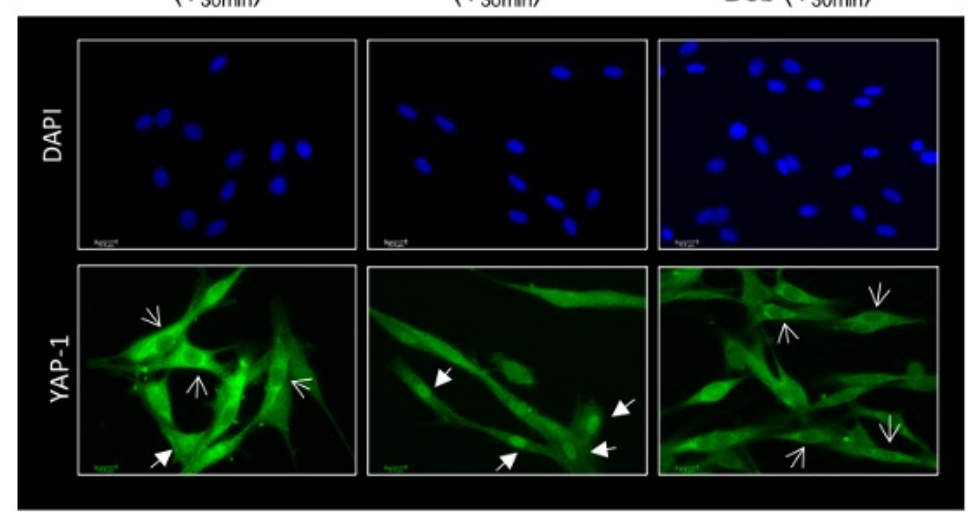

WM46 $\Delta$ ZIP9
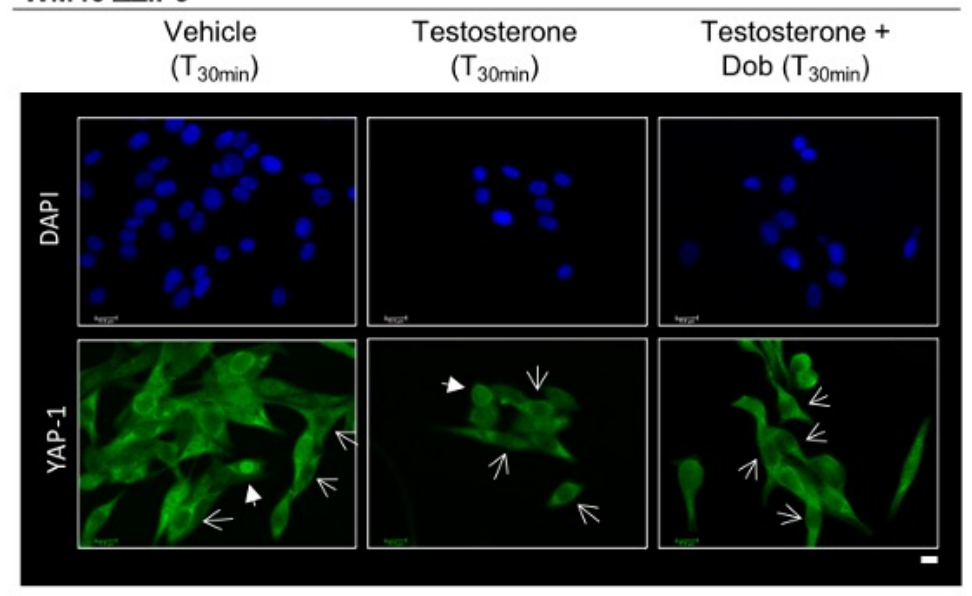

858 Fig. S5. A. Western blot showing baseline levels of ERK phosphorylation in wtZIP9 and $\Delta$ ZIP9

859 WM46 cells. B. Representative images of YAP-1 immunodetection after 30 minutes of exposure

860 to $100 \mathrm{nM}$ testosterone (T) $+/-8 \mu \mathrm{M}$ dobutamine (Dob) in wtZIP9 and $\Delta$ ZIP9 cells. The

861 percentage of cells with nuclear YAP-1 (per field) are shown in the upper-left side of each panel.

862 YAP1 is shown in green; $\beta$-actin is shown in red; DAPI highlights nuclear DNA in blue. Thin 
863 arrows indicate cells where YAP1 is not localized inside the nucleus. Opaque arrows indicate

864 cells with nuclear YAP-1. Scale bar $=15 \mu \mathrm{m}$. C. Percentage of WM46 wtZIP9 and $\Delta$ ZIP9 cells

865 transduced with pRRL empty vector (E.V.) or pRRL_hZIP9 expressing vector with nuclear

866 localization of YAP1. Cells were treated with vehicle and $100 \mathrm{nM}$ testosterone.

$867 * * * \mathrm{p}$ value $\leq 0.001 ; \mathrm{n} . \mathrm{s}>0.05$

868

869

870

871 


\section{Supplementary Figure 6}

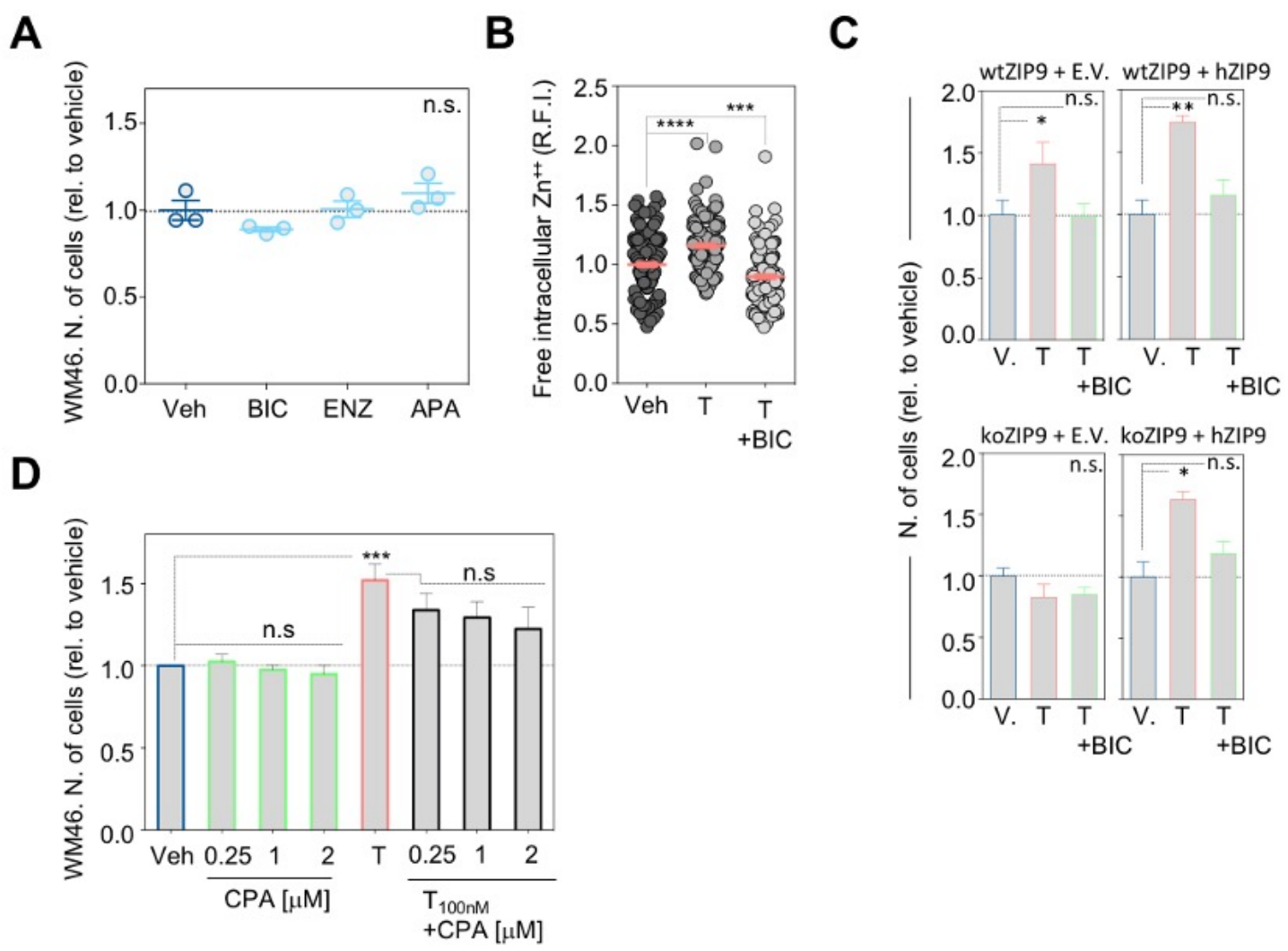

873 Fig. S6. A. Relative cell proliferation (cell number) in human-derived melanoma cells (WM46)

874 treated with $2 \mu \mathrm{M}$ AR inhibitors (BIC:Bicalutamide; ENZ:Enzalutamide; APA:Apalutamide). B.

875 Intracellular $\mathrm{Zn}^{++}$in WM46 cells treated with $100 \mathrm{nM}$ testosterone and/or $2 \mu \mathrm{M}$ bicalutamide

876 (BIC). C. Relative cell proliferation (cell number) of WM46 wtZIP9 and $\Delta$ ZIP9 transduced with

877 pRRL empty vector (E.V.) or pRRL_hZIP9 expressing vector. Cells were treated with vehicle

878 (V) or $100 \mathrm{nM}$ testosterone $+/$ - bicalutamide $(2 \mu \mathrm{M})$ D. Relative cell proliferation of WM46 cells

879 treated with 100nM testosterone and/or cyproterone acetate (CPA) for 6 days.

$* * * * \mathrm{p}$ value $\leq 0.0001 ; * * * \mathrm{p}$ value $\leq 0.001 ; * * \mathrm{p}$ value $\leq 0.01 ; * \mathrm{p}$ value $\leq 0.05 ; \mathrm{n} . \mathrm{s}>0.05$. 


\section{Supplementary Figure 7}

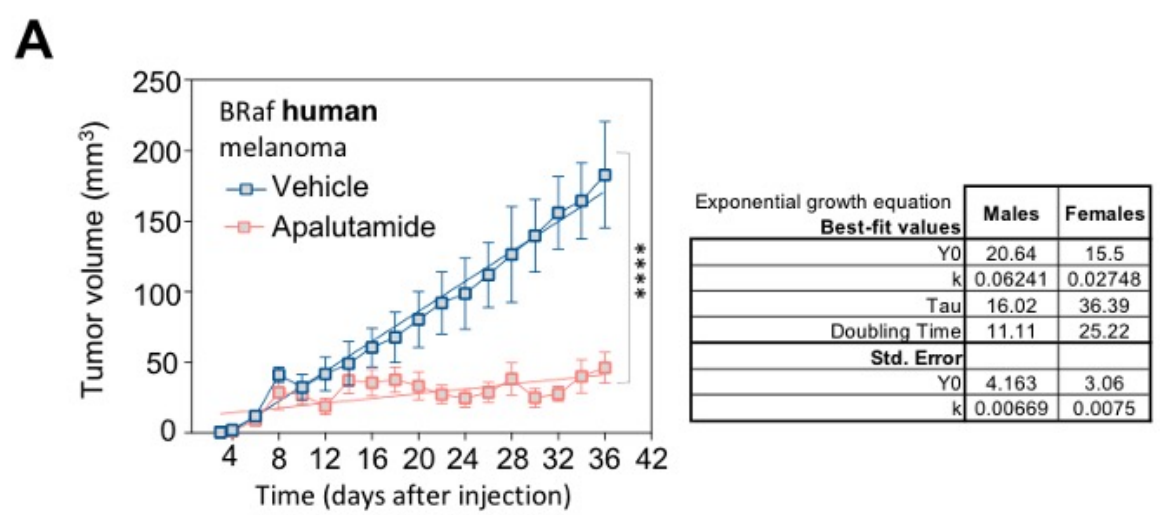

B

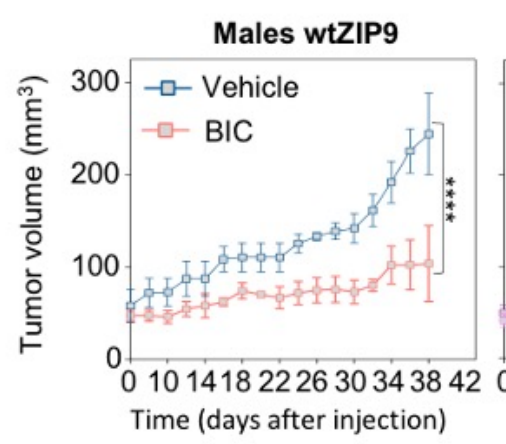

Females wtZIP9

C
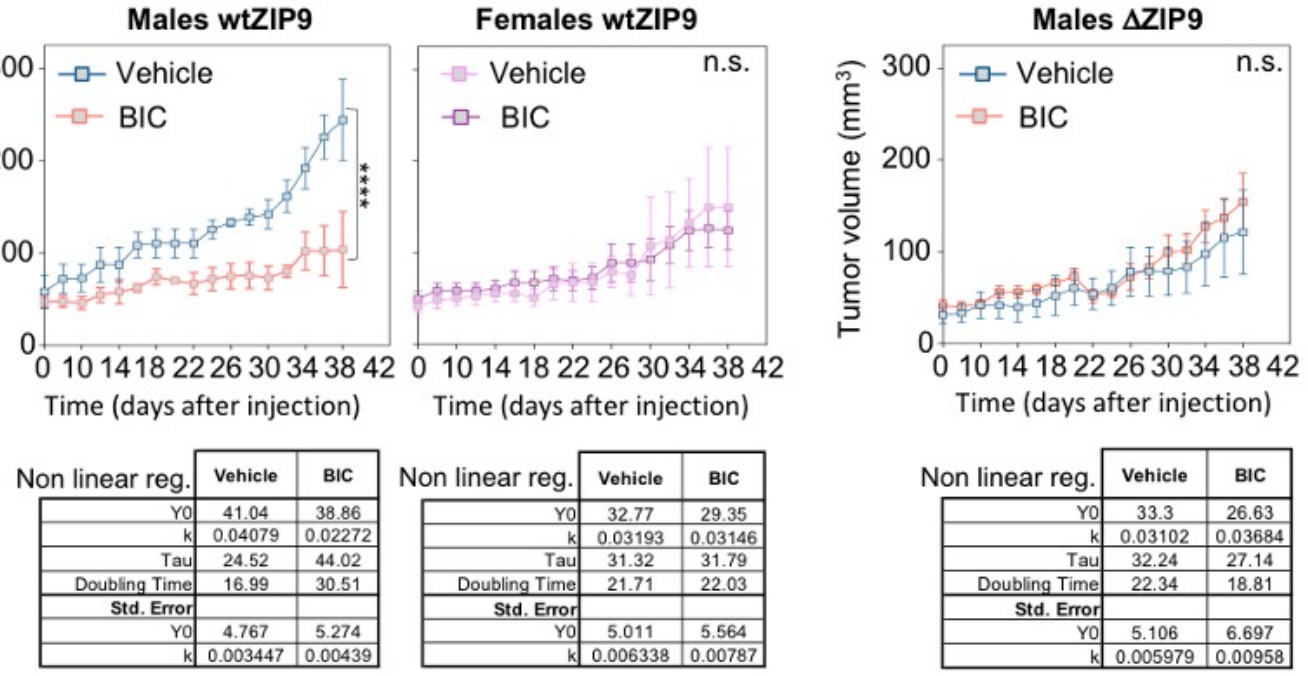

883 Fig. S7. A. Non-lineal regression analysis (Exponential fit) for males treated with vehicle vs.

884 APA. Doubling time for tumor growth: Vehicle treated males=16.0 days and APA treated

885 males $=36.4$ days. Linear regression analysis for slope comparison demonstrate significant differences between vehicle treated and apalutamide treated males ( $p$-value $<0.0001)$. B. Tumor

887 growth in mice bearing WM46 derived subcutaneous tumors. Daily treatment with bicalutamide

888 (30 mg/kg/day, via oral gavage) or vehicle are shown for both male and female mice. Linear regression analysis of slopes demonstrates significant differences between vehicle treated and 
890 Bic treated males ( $\mathrm{p}$-value $<0.0001$ ). C. Tumor growth in SCID male mice bearing $\triangle$ ZIP9 WM46

891 melanoma. Mice were treated daily with bicalutamide $(30 \mathrm{mg} / \mathrm{kg} / \mathrm{day})$ or vehicle via oral gavage.

892 Exponential fit analyses are included bellow each tumor growth graphical representation.

$893 * * * * \mathrm{p}$ value $\leq 0.0001 ; \mathrm{n} . \mathrm{s}>0.05$.

894

895

896

897

898

899

900

901

902

903 


\section{Supplementary Figure 8}

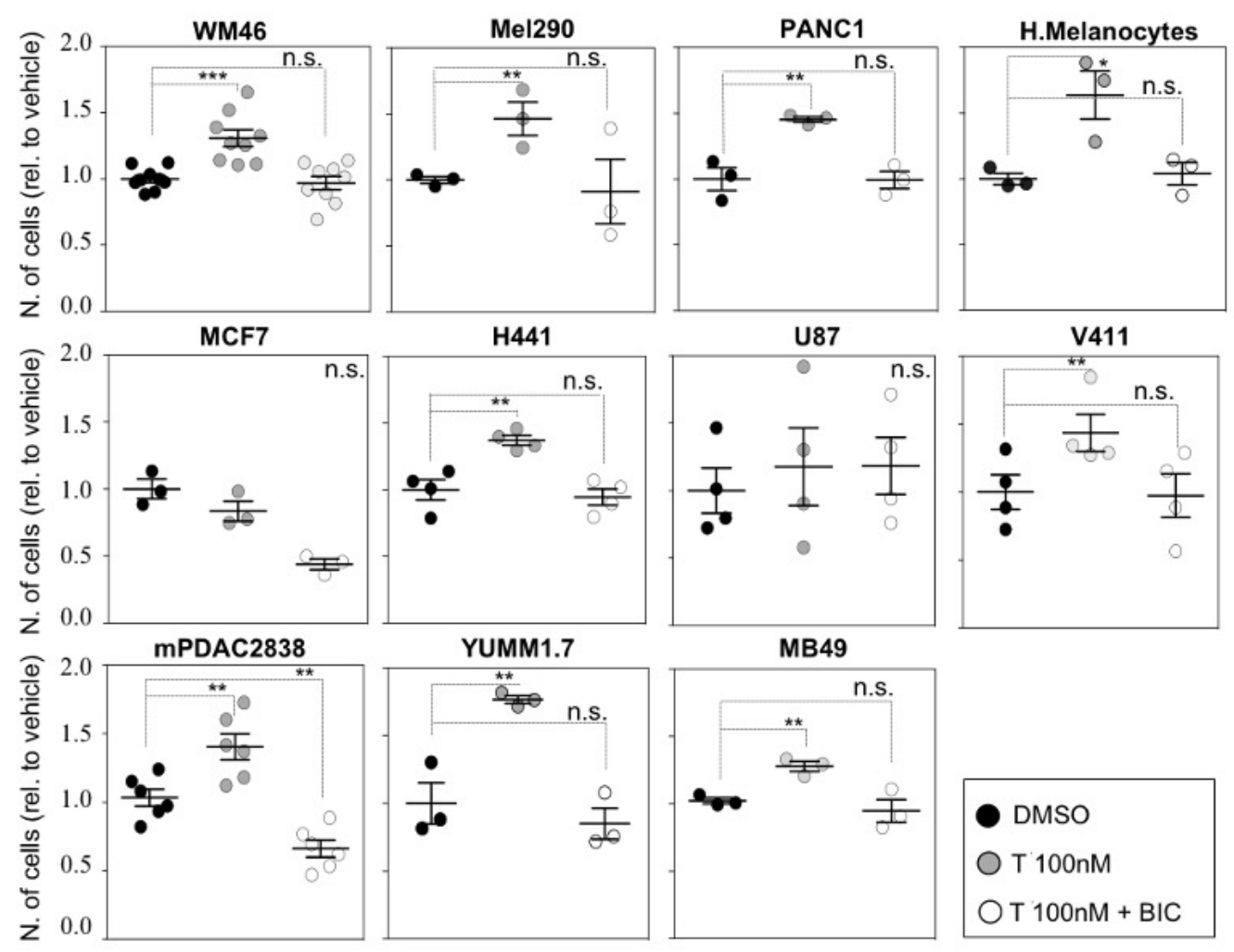

\section{Cell types}

WM46: Human skin melanoma Mel290: Human uveal melanoma YUMMS1.7: Murine skin melanoma PANC1: Human pancreatic adenocarcionma mPDAC: Murine pancreatic adenocarcinoma

MCF7: Human breast cancer MB49: Mouse bladder cancer H441: Human lung adenocarcionma U87: Human glioblastoma

V411: Human adult acute myeloid leukemia

Fig. S8. Relative cell proliferation of a battery of cell lines derived from diverse tumor types in response to $100 \mathrm{nM}$ testosterone $+/$ - bicalutamide treatment $(2 \mu \mathrm{M}) .{ }^{* * *}$ p value $\leq 0.001$; $* * \mathrm{p}$ value $\leq 0.01 ; * \mathrm{p}$ value $\leq 0.05 ; \mathrm{n} . \mathrm{s}>0.05$ 